\title{
Goldoni e gli attori: una relazione di imprescindibile reciprocità
}

\author{
Elena Sala Di Felice
}

\section{Il triangolo armonioso, obbiettivo della Riforma}

L'attenzione degli studiosi della drammaturgia goldoniana si è orientata, in modo sempre più deciso, verso la riflessione sul rapporto di stretta interdipendenza tra l'autore e gli attori, in funzione della Riforma e della conquista del successo. ${ }^{1}$ Questi argomenti sono stati dibattuti con particolare frequenza nei convegni e nelle pubblicazioni celebrative del recente bicentenario della

1. E’ ancora utile il lavoro di Attilio Gentile, Carlo Goldoni e gli attori, Trieste, Cappelli, 1951. Nel suo intervento al convegno del 1957 Mario Baratto sottolineava l'interesse specifico di Goldoni per le doti degli attori (cfr. "Mondo e teatro" nella poetica del Goldoni, in ID., Tre studi sul teatro, Venezia, Neri Pozza, 1964, poi 1971, p. 157-234 e in particolare p. 162-164); significative osservazioni si leggono poi, a proposito del contributo degli attori alle invenzioni del commediografo, nelle sue letture goldoniane in ID., La letteratura teatrale del Settecento in Italia, Vicenza, Neri Pozza, 1985. Sull'argomento ancora cfr. Franco FIDO, Guida a Goldoni. Teatro e società nel Settecento, Torino, Einaudi, 1977, poi 1987; ID., Goldoni e il linguaggio teatrale del Settecento, in ID., Il paradiso dei buoni compagni. Capitoli di storia letteraria veneta, Padova, Antenore, 1988, specialmente La parola degli istrioni: Goldoni e le maschere della Commedia dell'Arte, p. 149-164. Attento all'intreccio dei rapporti tra autore, attori e pubblico si è mostrato Cesare DE MiCHELIS, Carlo Goldoni e la professione di "scrittor di commedie», in ID., Letterati e lettori nel Settecento veneziano, Firenze, Olschki, 1979, p. 153-201. Ludovico Zorzi ha affrontato la questione della Riforma in rapporto alla persistenza di suggestioni derivate dalla Commedia dell'Arte nei saggi raccolti in L'attore, la commedia, il drammaturgo, Torino, Einaudi, 1990, cfr. la parte Verso il drammaturgo. Goldoni, p. 223 e ss. Specifico è lo studio di Carmelo AlBerTi, La scena veneziana nell'età di Goldoni, Roma, Bulzoni, 1990. Un'idea ben più complessa e mossa di quella tradizionale, della dialettica tra "mondo» e «teatro» emerge dal lavoro di Arnaldo Momo, La carriera delle maschere nel teatro di Goldoni, Chiari, Gozzi, Venezia, Marsilio, 1992, cfr. le p. 15-163. Per un orientamento generale, ma attento ai problemi dello spettacolo e dell'attore si consigliano: Siro FERrone, Carlo Goldoni.Vita, opere, messinscena, Firenze, Sansoni, 1990, con bibliografia ragionata e l'antologia della critica curata ed intelligentemente introdotta da Marzia PIERI, Il teatro di Goldoni, Bologna, Il Mulino, 1993. Di altri contributi più recenti, legati alla ricorrenza del centenario della morte darò notizia nel corso del lavoro; ma segnalo fin da ora lo studio di Franco VAzzOLER, Dramaturgie de l'auteur, dramaturgie des acteurs: le problème fondamental dans le théâtre de Goldoni, in Goldoni, le livre, la scène, l'image (Atti del Colloque alla Université de la Sorbonne Nouvelle, Paris III, 6 e 7 dicembre 1993), in "Chroniques Italiennes», n. 38, 1994, p. 51-67, le cui linee essenziali sono state riprese in Qualche (modesta) proposta sul libro del teatro, in Goldoni 1793-1993, Atti del Convegno del Bicentenario (Venezia, 11-13 aprole 1994) a c. di C.Alberti e G.Pizzamiglio, Venezia, Regione del Veneto, 1995, p. $157-160$. 
morte del commediografo; ${ }^{2}$ ma la questione risultava già ben rilevata nelle generose autotestimonianze di uno scrittore presto vocato al racconto autobiografico e alla riflessione critica sul proprio operato. In molte occasioni egli ha attestato la propria stima per gli indispensabili collaboratori e alleati nella Riforma del teatro e nella riconquista del pubblico, che doveva trovare nello spettacolo rinnovato non solo uno svago triviale, ma un'occasione di conoscenza e di educazione. I riconoscimenti tributati ai comici distinguono Goldoni dai letterati che, pur auspicando la moralizzazione e una maggiore dignità formale della scena, non avevano mai tralasciato di segnare le distanze fra la propria superiorità di «intellettuali»" ${ }^{3}$ e la condizione degli ignoranti istrioni; così essi avevano definito, con formula ricorrente, gli attori, cioè i «lavoratori», le "genti meccaniche» — se così si può dire- dello spettacolo. Di contro, con vivo senso realistico e senza sterile boria, il drammaturgo, presentando I due gemelli veneziani, una delle commedie della sua prima stagione, riconosceva che:

... la Commedia è Poesia da rappresentarsi, e non è difetto suo che ella esiga, per riuscir perfettamente, de' bravi Comici che la rappresentino, animando le parole col buon garbo d'un'azione confacevole;... (A, II, 156) ${ }^{4}$

2. E' indubbiamente significativo il fatto che, in occasione del centenario, la rivista della «Comédie française. Les cahiers» (n. 6, 1993) abbia dedicato una sezione a Goldoni et ses acteurs.

3. Il rapporto tra drammaturghi e professionisti della scena fu tutt'altro che pacifico nel corso del primo Settecento; anche i letterati, fautori per un certo periodo della loro vita dei teatri pubblici, come luogo di necessaria verifica per le opere destinate alla rappresentazione, come Pier Jacopo Martello (cfr. Della tragedia antica e moderna, in ID., Scritti critici e satirici, a c. di Hannibal S. Noce, Bari, Laterza, 1963, p. 203) e Scipione Maffei (cfr. Istoria del teatro e difesa di esso, in ID., De' teatri antichi e moderni e altri scritti teatrali, a c. di Laura Sannia Nowé, Mucchi, Modena 1988, cfr. le p. 33-34), si dichiararono poi risolutamente contrari, per ragioni moralistiche non meno che per sfiducia nelle capacità intellettuali ed artistiche dei comici, all'esibizione dei testi d'autore nei teatri venali, frequentati da un pubblico inetto ad apprezzarli. Martello scriveva: «Lontane dunque dal popolo le nostre commedie. Né la mia potrà certamente rappresentarsi che da un seminario o da un'accademia ad un'udienza scelta e raccolta, la maggior parte di letterati..." All'Eccellenza di Giovanbattista Recanati, nobile veneto fra gli Arcadi Teleste Ciparissiano. Lettera dedicatoria della commedia Che bei pazzi (1723), in Teatro, cit., vol. I, p. 236). Maffei, ingrato e dimentico del fatto che la sua Merope doveva in larga parte il successo all'abilità di Flaminia e Lelio, cioè i coniugi Riccoboni, dichiarava che "per rimettere la Tragedia e la buona Commedia in Italia, converrebbe poter bandire i Comici di professione e ridur le recite a compagnie di costumati e ben nati giovani» (Recensione a Pietro CALEPIO, Paragone della poesia tragica d'Italia con quella di Francia, Zurigo, Rordorf, 1732; poi Venezia, Zatta, 1770, nel vol. cit., p. 56; cfr. anche il Proemio all'ultima edizione della Merope curata dall'autore, Verona, Ramanzini, 1745, ivi, p. 75).

4. Citerò da Tutte le opere di Carlo Goldoni, a c. di Giuseppe Ortolani, Milano, Mondadori, 1935-56, voll. 14, indicando, tra parentesi dopo ogni citazione, il volume e la pagina. Con $\mathrm{A}$, seguito dal titolo della commedia, indicherò le prefazioni «L'Autore a chi legge»; con $\mathrm{M}$, seguito dall'indicazione della parte, del capitolo e della pagina, i Mémoires; con PP, seguiti dalla cifra romana indicante il tomo, e araba la pagina indicherò le Prefazioni Pasquali. Le scritture autobiografiche si leggono nel vol. I della citata edizione. Per le commedie indicherò l'atto, la scena; il volume e la pagina. 
Nella tarda autobiografia era riconosciuto all'abilità di Antonio Sacchi, un attore pur assolutamente restio a lasciarsi coinvolgere nel nuovo metodo di recitare, il successo della Notte critica, ossia i centoquattro avvenimenti in una medesima notte, un lavoro definito l'éprenve des Comédiens, tanto da miseramente cadere in occasione di una recita di dilettanti.

L'incrocio, nella memoria, dei contrastanti esiti delle realizzazioni, permetteva a Goldoni di enunciare la propria idea sui rapporti degli autori con gli attori, che tuttavia si deve leggere con la consapevolezza degli effetti prodotti dal «senno di poi»:

Cela prouve que la réputation d'un Auteur dépend souvent de l'exécution des Acteurs. Il ne faut pas se dissimuler cette vérité, nous avons besoin les uns des autres, nous devons nous aimer, nous devons nous estimer réciproquement, servatis servandis. (M, I, XLI, 191)

Sarebbe fin troppo facile accumulare altre dichiarazioni dello stesso tenore, pertanto si ricorderanno pochi passi per avviare la riflessione ripercorrendo qualcuna delle tappe più significative della questione che Franco Vazzoler ha definito limpidamente come «la drammaturgia degli attori»:

Credo che il rapporto con gli attori costituisca un capitolo del 'libro del teatro' che oggi merita di essere riletto con strumenti diversi da quelli della semplice analisi letteraria [...] credo che sia importante rifarsi all'attore come 'questione centrale' (o come 'centro della questione'), [...] Bisogna [...] riferirsi alla drammaturgia goldoniana come ad una drammaturgia d'autore che si pone in rapporto alla commedia dell'arte intesa non tanto, convenzionalmente, come teatro delle maschere, dell'improvvisazione ecc., ma come 'drammaturgia degli attori'. 5

Per quanto cauti di fronte alle testimonianze d'autore, dobbiamo notare la franchezza del linguaggio e l'importanza del contenuto di certi passi dell'autobiografia, dove l'anziano esule rievoca, usando il tempo presente, in funzione icastica, quasi d'ipotiposi, l'ancor giovane drammaturgo, di ritorno a Venezia con la compagnia Imer, cui si era appena aggregato:

Je suis avec les Comédiens comme un Artiste dans son attelier. Ce sont d'honnêtes gens, beaucoup plus estimables que les esclaves de l'orgeuil et de l'ambition. (M, I, XXXV, 160)

Non meno notevoli sono i cenni alla specifica cultura degli attori, in particolare quelli sul Truffaldino Sacchi; un improvvisatore della imagination vive et brillante, le cui subitanee trovate erano sempre - secondo quanto ricordava Goldoni- pertinenti au fond de la scene; le cui battute non deriva- 
vano né dal comune patrimonio dei Comici dell'Arte, né dal linguaggio del popolo; esse erano invece sostenute da conoscenze letterarie e filosofiche ricche, ma piegate da quella superiore intelligenza «creativa» al servizio della comicità più comunicativa: Seneca, Cicerone, Montaigne erano citati dall'improvvisatore in modo da riuscire comprensibili à la simplicité $d u$ balourd e da suscitare irresistibilmente il riso. ${ }^{6}$

Leggiamo anche nel Teatro comico chiari riferimenti ad un sapere proprio dei teatranti: a Tonino è assegnata una battuta nella quale egli si mostra preoccupato che le sue abilità, conseguite con lo studio segondo l'arte, possano essere compromesse dalle innovazioni delle commedie "di carattere», "premeditate», rendendo lui, pur sperimentato professionista, insicuro dei propri mezzi. 7

La conferma della consapevolezza degli attori — che presuppone l'accordo del commediografo - circa il valore delle loro conoscenze, si deduce dalle battute di Anselmo-Brighella al poetastro Lelio: lo Zanni non solo rivendica, al di sotto della maschera, la propria dignità, ma dichiara indispensabile, per la resa di qualsiasi carattere, la competenza intellettuale dell'attore professionista:

... son un omo che, se non è poeta per l'invenzion, ha però quel discernimento che basta per intender el so mistier. Un comico ignorante no pol riuscir in nissun carattere. (II, $1 ;$ II, 1067) ${ }^{8}$

Ancora nella stessa commedia l'autore affidava al proprio portavoce, il capo-comico Orazio, una positiva valutazione morale del mondo del teatro:

In tutta l'arte comica vi saranno pur troppo de' malviventi; ma di questi il mondo è pieno, e in tutte le arti qualcheduno se ne ritrova. Il comico deve essere, come tutti gli altri, onorato; deve conoscere il suo dovere, e deve essere amante dell'onore e di tutte le morali virtù. (I, 6; II, 1056)

E' interessante osservare che l'immagine del mondo del teatro, in chiave proprio moralisticamente positiva, appare nella dedica - pubblicata nella stampa Bettinelli (1750) — ad Antonio Condulmer dei Due Gemelli veneziani (1747). Scritto, questo, rivelatore delle esitazioni con cui l'avvocato aveva abbandonato - a Pisa- l'onorevole professione forense per dedicarsi a quella più precaria e socialmente almeno "dubbia» di "scrittore di commedie»:

6. Cfr. M, I, XLI, p. 190-191.

7. Il teatro comico, I, 3; II, 1053.

8. Letta «in situazione», cioè nella precisa circostanza di enunciazione, la battuta darebbe ragione a Franco Vazzoler: «... bisogna pensare gli attori (e Goldoni, Chiari e Gozzi hanno, almeno qualche volta, dimostrato di saperlo) come gli eredi di un grande sapere teatrale, diverso da quello dei letterati..." (Qualche (modesta) proposta..., cit. p. 159) 
Era in concetto di scandaloso il Comico Teatro [...] Ma quando ho veduto che le persone nobili, di dottrina, di senno, di esemplari costumi e di grado cospicuo, hanno creduto degno di sé l'onesto divertimento delle mie Commedie [...] ho preso animo e lena, e [...] liberatomi da ogni sorta di scrupolo, mi sono intieramente, e con animo quieto e tranquillo, alla intrapresa mia professione abbandonato. (II, 149)

Questa «confessione» è di tre anni posteriore all'esibizione, da parte della troupe Medebach della prima pièce composta da Goldoni ad istanza del Pantalone D'Arbes. In questo lasso di tempo l'autore s'impegnò con fervore al rinnovamento del teatro, svolgendo una doppia attività "pedagogica»: egli, educando gli attori al proprio "metodo" di recitazione, si proponeva di elevare anche i gusti del pubblico, che aveva già peraltro scoperto disgustato dalle sconce arlecchinate. Ci sembra di poter asserire che il commediografo —ormai modernamente deciso nella scelta della propria identità professionale- intese costruire con assiduità e lucida intelligenza quello che si vorrebbe chiamare un triangolo comunicativo che legava in nuova armonia l'autore e il pubblico, attraverso la mediazione degli attori che dovevano diventare responsabilmente collaborativi.

L'azione riformatrice di Goldoni è stata interpretata un po' diversamente da Ginette Herry, una studiosa specialmente attenta ai rapporti del commediografo con le persone oltre che con la professionalità e le abilità degli attori; rapporti da lei ripercorsi tanto sul filo delle testimonianze dirette, autobiografiche, quanto sulle tracce rilevate «in filigrana» nella tessitura delle commedie. La studiosa ha preso le mosse dalle critiche alle regole dei commedianti, illustrate da Gaetano Casali, che avrebbero suggerito all'autore la scelta di una contrerègle fondata sulle doti degli interpreti, la cui collaborazione avrebbe però dovuto, alla fine, ritorcersi contro i loro interessi:

La «réforme» cherche en effet à les [gli attori] amener, dans leur dire et leur jeu, à se mettre au service d'un auteur et à renoncer donc à leur rapport direct et autonome avec le public.?

Il tentativo complesso di sfruttare per le proprie invenzioni le doti dei singoli, pur senza "prendere di petto" le convenzioni — pur giudicate irragionevolmente dannose al teatro- non poteva muovere che dalla «drammaturgia degli attori», non già per aggirarla, bensì per trarne possibile profitto.

9. Ginette Herry, De Teodora à Maddalena in "Comédie française...", cit., p. 15-27. La citazione è a p. 15. Della stessa studiosa va ricordato il saggio su "Il teatro comico» $o$ il prezzo della riforma, in "Studi goldoniani», n. 4, 1976, p. 7-47. Vi si presta intelligente attenzione alle questioni economico-sociali del teatro, che incidevano sul progetto riformistico di Goldoni nel contesto del mercato teatrale di Venezia e all'interno della «società» dei commedianti lavoratori, del cui profitto l'impresario capocomico era responsabile. (p. 13-14) 
Infine resta particolarmente significativo il fatto che il passo citato dalla dedica sia stato scritto proprio nello stesso anno $1750,{ }^{10}$ segnato dalla composizione della commedia programmatica Il teatro comico, nonché dalla matura decisione di Goldoni di affermare, con la stampa Bettinelli, la propria identità di letterato, capace di consegnare alla posterità ciò che fino a quel momento era stato destinato all'effimero del teatro, alla "consumazione» dell'esecuzione attoriale e della fruizione da parte del pubblico, condizione della cui difficoltà la Herry ha rintracciato le spie nelle pieghe, spesso dolorose, della commedia-prefazione. ${ }^{11}$

\section{Pantalone: dalla maschera al volto}

La propria storia, che Goldoni ha più di una volta dichiarato di aver ricostruita come storia del proprio teatro, è stata scandita secondo le tappe dei suoi incontri con gli attori che ne hanno sollecitato le capacità inventive, promuovendo la riflessiva osservazione delle loro specifiche attitudini tanto intellettuali e psicologiche quanto fisiche e corporee. L'incontro, e l'amicizia — fin dal 1733 — con Gaetano Casali valse all'aspirante commediografo la scoperta che le regole dei commedianti non erano meno insulse e stravaganti, ridicole di quelle dominanti nel teatro per musica; ${ }^{12}$ ma non meno significativi sono i ricordi tramandati nelle stesse Prefazioni Pasquali intorno agli atteggiamenti, alle posture che quel pur eccellente attore assumeva involontariamente sulla scena e che lo rendevano inadeguato - agli occhi attenti di Goldoni- per il ruolo di secondo Amoroso, che quegli rivestiva nella compagnia Imer, al teatro San Samuele:

Una certa serietà nel sembiante, una certa durezza nella persona, un'inclinazione involontaria del fianco e della spalla verso il Personaggio con cui recitava, lo facevano scomparire, malgrado le belle cose ch'egli diceva: all'incontro nelle Tragedie riusciva mirabilmente, e soprattutto nelle parti gravi... (PP, XIII, 713)

Questa affermazione appartiene ad un'epoca tarda, tuttavia sembra veramente affidabile perché non fa che ribadire quanto si legge nella prefazione della Castalda, commedia andata in scena nel 1751, e stampata nel 1755, quando era da poco in corso l'esperienza del teatro di San Luca, che sappiamo essere stata ben diversa da quella del Sant'Angelo proprio perché mancavano l'intesa, la coesione e il comune intento innovativo che avevano animato la troupe del Medebach. Queste circostanze più difficili motivano l'enfasi che

10. Sulla coincidenza intorno alla data del 1750 di tanti eventi importanti ha richiamato l'attenzione C. De Michelis, (Carlo Goldoni e la professione... cit., p. 154-55).

11. Cfr. G. Herry, «Il teatro comico»... cit., p. 22: «il trionfalismo e il volontarismo ricorrenti nelle battute riformatrici ricoprono una realtà piena di nodi difficoltosi, nella quale operano le procedure drammaturgiche».

12. Cfr. PP, XI, 693. 
cade sulla fermezza dell'autore nel mantenere saldo il proprio metodo da un lato; dall'altro sulla dichiarazione di utilità di ogni attore, quale che ne fosse il ruolo, come se il drammaturgo volesse assicurare, con le parole qui riportate, a tutti i suoi nuovi collaboratori attenzione, apprezzamento, volontà di valorizzazione:

Io ebbi sempre nello scrivere, ed ho tuttavia, un precetto asprissimo, che gli altri Scrittori per lo passato non hanno avuto, quello cioè di adattare la Commedia alla compagnia degli Attori, e non poterli scegliere per la rappresentazione delle Opere mie. Da ciò ne avviene, che conosciuto da me il valore d'un Personaggio, rade volte m'inganno, e poco felici riescono alcune scene, quando incerto sono di chi le debba rappresentare. Per me nessun Personaggio è inutile. Ciascheduno ha qualche carattere particolare, che può servire al Teatro... (IV, 7)

Il Casali fu oggetto di un'osservazione dalla quale scaturì il carattere del padre di Griselda, povero ma virtuoso e dignitoso nella fortuna come nell'umiliazione, inserito ex novo nella rielaborazione del melodramma zeniano:

J'avois imaginé ce nouveau personnage pour donner un rôle à mon ami Casali: cette épisode donna un air de nouveauté à la Tragédie, la rendit plus intéressante, et me fit passer pour Auteur de la Pièce. (M, I, XXXVII, 168)

L'insistenza sugli elementi patetici, connessa alla specifica abilità dell'interprete, lascia intendere come l'autore si fosse applicato a far convergere verso il successo i due elementi costitutivi della sua drammaturgia: l'autocoscienza del proprio valore di autore, mai disgiunta però dalla consapevolezza che il testo da lui ideato era destinato alla recitazione, cioè ad essere interpretato (ecco l'altro elemento) da un attore e per un pubblico di spettatori che, in questo caso, avrebbe dovuto provare piacere nel commuoversi fino al pianto. 13

Goldoni, riconoscendo il merito degli attori che avevano portato al successo i suoi componimenti, si attribuisce una capacità di preveggenza di tale risultato; analogamente anticipa il disegno della Riforma, che fu invece il frutto di prolungate e diversificate esperienze. Ricordando il rimpasto della compagnia Imer nella quaresima del 1738, lo scrittore ribadiva —ancora ricorrendo alla vivacità del presente storico- gli incoraggiamenti che allora avrebbe rivolto a se stesso, incitandosi ad osare l'attuazione dell'ambizioso progetto proprio fidando nella disponibilità e bravura dei nuovi interpreti, che gli avrebbero offerto gli spunti per le sue innovazioni: le commedie di carattere, sull'esempio eccellente di autori come Molière, come gli antichi: des larmes au XVIIe siècle, in "Littérature», n. 9, febbraio 1973, p. 56-73. 
Me voilà, me disois-je à moi-même, me voilà à mon aise; je puis donner l'essor à mon imagination [...] j'ai des Acteurs qui promettent beaucoup [...] Allons, continuois-je dans mes réflexions; voici le moment peut-être d'essayer cette réforme que j'ai en vue depuis si long-tems. (M, I, XL, 184)

Nella ricerca dell'attore, capace di soddisfare le proprie esigenze, e - stando a quanto tramandano i Mémoires - l'ansia di rinnovamento del commediografo, questi avrebbe «incrociato» quella dell'attore, il Pantalone Golinetti, disponibile ad abbandonare la maschera per sperimentare un «carattere» al posto del «tipo»:

Je m'arrêtai au Pantalon Golinetti, non pas pour l'employer avec un masque qui cache la phisionomie, et empêche que l'Acteur sensible fasse paroître sur son visage la passion qui l'anime; mais je faisois grand cas de sa manière d'être dans les sociétés où je l'avois vu et sondé... (ivi, 185)

All'inizio della carriera Goldoni incontrò degli attori che interpretavano il personaggio tradizionale del vecchio mercante, avaro e suscettibile al fascino femminile oltre quanto fosse consono alla grave età. Ma quegli stessi Pantaloni rivelarono al commediografo, attraverso le qualità, l'indole personale, le doti espressive e gestuali, il complesso del loro professionismo attorico, altre risorse del personaggio, suscettibile di trasformarsi in "carattere» con una mutazione che avrebbe necessariamente coinvolto il «mestiere» dei loro compagni.

L'interesse di Goldoni era molteplice: concerneva in toto la personalità dell'interprete, la sua intelligenza, il suo fisico, studiati con una partecipazione del tutto priva di ogni pretesa di superiorità.

Il desiderio di trarre il massimo profitto dagli atteggiamenti involontari di Casali aveva rivelato al drammaturgo la feconda risorsa del patetico; ora le doti di brillante simpatia del Golinetti lo indussero a proporre quel Pantalone a volto scoperto nelle vesti del Cortesan. ${ }^{15}$ Era l'inizio da una parte del nuovo modo di essere dell'attore, ben più personalmente coinvolto nella recitazione, cui la sua mimica facciale avrebbe dovuto contribuire significativamente; dall'altra parte si trattava della «redenzione» della maschera-tipo dai suoi tradizionali vizi, sostituiti con le ideali virtù del buon cittadino, difensore dell'onore della patria, dell'onestà, del lavoro, e finanche della borghese «morale di famiglia».

14. Il Gentile riporta a confronto la composizione della compagnia Imer nel 1734, e quella del 1738 (cfr. Carlo Goldoni e gli attori..., cit., p. 8). Nelle due formazioni erano presenti interpreti che, a vario titolo, segnarono l'esperienza dell'autore. Il Gandini, Brighella trasformista, che nel 1738 lasciò l'Imer, fu interprete, caricaturalmente efficace, di anziani personaggi femminili al San Luca; dove il Goldoni lavorò anche con il Majani, passato dai ruoli comici a quelli di primo Amoroso. Nuovi «acquisti» furono, in occasione di quel «rimpasto», il Golinetti e il Sacchi.

15. Franco Vazzoler indica un caso emblematico di convergenza nella disponibilità alla sperimentazione di autore e attori nel desiderio di rinunciare alla fissità della maschera di interpreti come Golinetti, D’Arbes, Collalto (cfr. Qualche (modesta) proposta ... cit., p. 160). 
Dunque all'origine d'un carattere, che si voleva esaltare rispetto alla maschera, si può scorgere lo stimolo proveniente dalle doti personalissime di un attore, che dovevano presto interagire con l'apporto di un altro genere di osservazioni altrettanto empiriche, il cui frutto Goldoni riversò nella sua Bancarotta, al cui concepimento - se crediamo alle sue asserzioni- avrebbe contribuito anche un intento morale. ${ }^{16}$

Dalle pagine dei Mémoires e dall'Autore a chi legge premessa alla stampa del 1757 , si può dedurre che la pièce abbia obbedito a varie sollecitazioni e osservato vari accorgimenti, della cui opportunità l'autore si era via via persuaso: egli era allora (1740) console della repubblica di Genova, carica che lo poneva a stretto contatto con il mondo mercantile, permettendogli di comprenderne i meccanismi economici tanto da proporli realisticamente in scena con intento didascalico; in secondo luogo lo sdoppiamento del personaggio del mercante nel padre disonesto, cui si contrappone il figlio Leandro, sollecito del proprio onore, ripeteva l'espediente, già posto in opera nel Momolo cortesan, e più tardi ridotto a precetto generale:

Pour qu'un caractere quelconque fasse plus d'effet sur la scene, j'ai cru qu'il falloit le mettre en contraste avec des caracteres opposés ... (M, I, XL, 185-86)

Commentando l'esito felice della recita, l'autore, al momento della stampa, giustificava i propri interventi, che avevano fatto diventare d'autore un lavoro originariamente scritto solo in parte. La circostanza era sfruttata per avvalorare la scelta definitiva di Goldoni di essere scrittore, esibendone le motivazioni strettamente teatrali, cioè l'esigenza di un compiuto equilibrio della recita, cui gli attori, affidandosi al solo mestiere, non potevano pervenire. Al tempo della prima rappresentazione, quantunque egli avesse -si direbbe come drammaturgo-regista- «bien concerté les Acteurs», le parti affidate all'improvvisazione non gli parvero in armonia con quelle scritte: "On n'y voyoit cette égalité de style qui caractérise les Auteurs» (ivi, 186), il quale pertanto richiedeva l'assunzione della compiuta autorità dello scrittore.

16. Arnaldo Momo ha notato in questo primo personaggio-carattere dei «tratti già realistici, in una Venezia dove magari predomina il 'colore' [...] ma che è già colta dal vero, con la sua vocazione allo sfruttamento del forestiero» (Dal «Teatro» al "Mondo» di Goldoni, in La carriera... cit., p. 47). L'invenzione del nuovo personaggio, appoggiato al Pantalone «smascherato" è un primo indizio della vocazione della maschera del mercante a diventare carattere tratto dall'osservazione empirica del «Mondo».

17. Nell'autobiografia si legge: «Initié par mon nouvel emploi dans la connoissance des Négocians, je n'entendois parler que de faillites» (M, I, XLII, 193). Egli poteva vedere come i bancarottieri coinvolgessero nella rovina e nel disonore anche gli onesti creditori, sì che prese spunto da tali fatti per una commedia rispettosa dell'antico precetto «castigat ridendo mores». Lo stesso intento moraleggiante è esibito nell'Autore a chi legge (I, 942). Sulla realtà della crisi economica che gravava sulla Repubblica specialmente negli anni 1741-45, cfr. Jean Georgelin, Venise au siècle des Lumières, Paris-La Haye, 1978. 
D'altro canto, già a proposito del rimpasto della compagnia Imer rievocandone il primo Amoroso, ${ }^{18}$ Goldoni rivelava come gli attori, pur di strappare un applauso personale, non esitassero ad inserire nella recita episodi o trovate sceniche incongrue rispetto al contesto. L'argomento doveva essere ripreso anche nelle presentazioni di rifacimenti di altre commedie, dove lo spazio riservato all'improvvisazione delle maschere era stato sostituito dalle battute dello scrittore, certo più rispettose del generale equilibrio.

La Bancarotta (1741) era la rielaborazione di un soggetto dell'Arte, come si dice nell'Autore a chi legge. ${ }^{19} \mathrm{La}$ circostanza merita qualche riflessione, sia come prova del rapporto di continuità tra la tradizione dell'Improvvisa e l'esperienza perennemente in fieri della Premeditata (per usare il lessico goldoniano), sia come stimolo a riflettere sulla complessità delle relazioni che andavano stabilendosi tra gli attori e l'autore, allora "principiante», e tutt'altro che già in possesso del progetto riformatore, al di là di quanto egli avrebbe affermato negli anni della maturità.

L'altro attore che consentì a Goldoni di chiarire anche a se stesso la propria vocazione e di mettere veramente alla prova le risorse della sua intelligenza teatrale, fu Cesare D’Arbes. Le pagine dei Mémoires in cui è narrata la visita

18. Cfr. PP, XIII: «Primo Amoroso in attuale esercizio Antonio Vitalba Padovano, comico il più brillante, il più vivo che siasi veduto sopra le Scene. Parlava bene e con una prontezza ammirabile, e niuno meglio di lui ha saputo, come dicono i Commedianti, giocar le Maschere [...]. Qualche volta però gli Arlecchini si dolevan di lui, perché scordandosi il carattere dell'Amoroso, faveva egli l'Arlecchino [...] Nelle scene più serie e più interessanti cercava di cavar la risata; e non esitava a rovinar la Commedia...» (p. 712-713). Vitalba non aveva esitato, pur di provocare il riso del pubblico, quando recitava la parte eroica di Belisario, ad usare il bastone, che gli doveva servire da appoggio in quanto il personaggio figurava cieco, per colpire una delle sue guardie (ibidem).

19. Su questa commedia cfr. Pietro SpezZANI, Per un'analisi critica e linguistica della "Bancarotta» di C. Goldoni, in «Studi Goldoniani», n. 4, 1976, p. 83-104; Piermario VesCovo, Per la storia della commedia cittadina veneziana pre-goldoniana, in Quaderni Veneti, n. 5, Ravenna, Longo, 1987, p. 37-80, che sviluppa l'indagine sul Mercante fallito di Tomaso Mondini (Venezia, Lovisa, 1693), da cui il nuovo commediografo avrebbe preso le mosse (cfr. specialmente le p. 58 e 62-64). Anche Norbert Jonard insiste sul rapporto tra la consapevolezza della crisi economica e l'assunzione di quel soggetto; cfr. la n. 3, p. 632 dell'ed. dei Mémoires, Paris, Aubier, 1993, che lo studioso ha corredato di commenti preziosi per i riferimenti alla storia; al teatro come istituzione e come letteratura; agli attori. E queste note contengono ancora precise, ricche informazioni bibliografico-critiche. La Bancarotta come noi la leggiamo comparve nel t. X dell'ed. Paperini, Firenze, 1752; essa è frutto di una rielaborazione che ha assorbito e messo a frutto l'esperienza — ormai conclusa - della collaborazione con la compagnia Medebach. La prefazione dichiarava di emendare il testo primitivo, solo parzialmente scritto: vale a dire che la commedia è stata rifatta nell'ottica della maturata Riforma (cfr. I, 944); e su questo è doveroso il richiamo alle osservazioni di Gianfranco FolENA, Una lingua per il teatro, in ID., L'Italiano in Europa, Torino, Einaudi, 1983, specialmente alle p.165-66. Più recentemente Arnaldo Momo è tornato a spiegare la necessità, avvertita da Goldoni, di rielaborare il testo delle prime pièces, necessariamente rispettose delle costrizioni cui si attenevano gli attori dell'Improvvisa, pronti a «rubarsi» l'un l'altro le battute, in gara per il successo e prima ancora per la difesa del proprio spazio nel contesto di una difficile, precaria "professione». (cfr. La carriera delle maschere... cit., p. 12-13) 
dell'attore nello studio pisano dell'avvocato, sorpreso in un momento di scontentezza, di delusione professionale, costituiscono un racconto che si può definire veramente metateatrale. Il personaggio seppe sedurre il suo ospite recitando con garbo la richiesta di una collaborazione, tanto che la sua gestualità, studiata per testimoniare la propria abilità — si accarezzava la rotondità del ventrerimase impressa nella memoria del commediografo, che assistette anche ad un vero e proprio elegante lazzo quando, ottenuta la promessa di una commedia, l'attore fece scivolare delle monete nella tabacchiera, fingendo - litoticamente- di voler celare il gesto, che invece veniva esibito garbatamente. ${ }^{20}$ Questo racconto metateatrale, di straordinaria efficacia, sembra dar ragione a Vazzoler quando sostiene che fu della più grande importanza per il rinnovamento del teatro italiano nel Settecento la convergenza delle disponibilità, delle necessità di trasformazione da parte degli attori non meno che di quell'autore:

...je crois que vraiment l'élan vers le renouveau, le bouleversement des rôles, la recherche de nouvelles possibilités pour leurs moyens expressifs ne réside pas seulement chez l'auteur Goldoni, mais aussi chez les acteurs... ${ }^{21}$

L'interazione drammaturgo-esecutori era già stata messa in relazione con l'esigenza-possibilità della riforma del teatro da un attore, Riccoboni, che aveva superato la propria avvertita inferiorità culturale, che aveva studiato ed era diventato un eccellente interprete, restauratore del repertorio tragico italiano «regolare», un autore di canovacci dove s'affacciava già proprio il «carattere». E infine si dimostrò un profondo conoscitore delle differenti tradizioni teatrali europee. Nel suo Discorso della Commedia all'improvviso egli lamentava la carenza, in Italia, di autori:

Comparischino de' Poeti ad esibire buone Comedie e vedrete a' Comici di poco talento sucedere de' valentuomini che di buona voglia entraranno in una proffessione che, se al presente gli fa orrore, a l'ora gli invogliarebbe dandogli speranza di guadagno e di riputazione. ${ }^{22}$

20. Cfr. M., I, LI, 229-230.

21. F. VAZZOLER, Dramaturgie de l'auteur... cit., p. 60. Anche Gentile aveva notato: «vennero a cercarlo i comici, presso i quali era ormai conosciuta la sua passione di ripulire il teatro e la sua abilità nel servirli» (Carlo Goldoni e gli attori... cit., p. 17). Quanto al magistero dell'autore, veramente drammaturgo-regista, si legge nello stesso libro che fu sua abilità «aiutarli [scil. gli attori] a scoprire in sé spiriti, finezze, capacità latenti nell'inerzia del mestiere...» (p. 4)

22. L. RiccobOni, Discorso della Commedia all'improvviso e scenari inediti, a c. di Irène Mamczarz, Milano, Il Polifilo, 1973, p. 12. Vazzoler ha ricordato l'apprezzamento dell'attore e studioso del teatro, Luigi Riccoboni, per la naturalezza della recitazione improvvisa, superiore a quella prevedibile da chi interpretasse una parte premeditata (Cfr. Dramaturgie de l'auteur... cit. p. 53 che rinvia a L. RICCOBONI, Histoire du théâtre Italien depuis la décadence de la Comédie latine, avec un catalogue des Tragédies et Comédies imprimées depuis l'an 1500 jusqu'à l'an 1600 et une dissertation sur la tragédie moderne, Paris, Dormel, 1728-1732, vol. I, p. 61-62). 
Il comportamento di D'Arbes sembra aver realizzato le previsioni del celebre Lelio: il Pantalone che richiedeva una commedia all'avvocato, a nome di tutta la compagnia Medebach, aveva abbandonato la propria famiglia onorata, davanti alla quale non avrebbe voluto ripresentarsi se non "couronné de laurier", che i suoi estimables talens potevano assicuragli, se impiegati adeguatamente nella recita di testi altrettanto meritevoli.

L'intreccio tutt'altro che schematizzabile - come si è fatto per troppo tempo - tra conservazione del patrimonio umano e culturale della Commedia dell'Arte e rinnovamento, si coglie proprio nella collaborazione tra D'Arbes, i compagni e l'ormai ex-avvocato, ingaggiato come poeta di una troupe capace di avvertire i limiti e le insufficienze del solo studio segondo l'arte. Ma non è di poco momento che il successo abbia pienamente coronato l'esecuzione da parte di D'Arbes dell'Uomo prudente; un altro canovaccio di cui Goldoni aveva questa volta scritto a Pisa, tra il 1747 e il 1748 , tutte le parti. Il pubblico avrebbe gradito quel complesso di romanzesche avventure, tra tentativi di avvelenamento e dibattito processuale, dove l'autore potrebbe aver sfoggiato anche le proprie competenze forensi. Ma vera innovazione poteva considerarsi l'inserimento nell'antico organismo di un carattere patetico, quello del Pantalone, trasformato in «riche et honorable négociant qui cherche à dissimuler les désordres d'une famille en crise». Ricorrendo persino a qualche inquinamento delle prove, il padre, interpretato magistralmente da D'Arbes, cioè Pantalone, oggetto dell'attentato, salva moglie e figlio colpevoli, e con suggestione emotiva convinceva loro alla resipiscenza, e il pubblico alle lacrime, e non più solo al riso. ${ }^{23}$ Grazie alle capacità attoriali specifiche del D'Arbes il commediografo scopriva una vena tematica che avrebbe poi sfruttato con abilità.

In quell'occasione egli compensava l'attore dell'insuccesso zdel Tonin Bellagrazia, di cui pure gli attori erano stati entusiasti alle prove e che invece non aveva convinto il pubblico; di quella caduta però l'autore - fedele alla propria idea di interazione armoniosa - non aveva incolpato né il pubblico, né gli esecutori: aveva preferito riconoscere le inadeguatezze di quella proposta scenica, per impegnarsi poi a far risaltare le doti di un attore in particolare, come prova il fatto che proprio al Pantalone sono riservati in quantità proporzionale molto maggiore gli interventi negli scambi conversazionali. A lui tocca informare il pubblico degli antefatti e orientarlo nel prossimo sviluppo

23. A proposito dell'Uomo prudente e del suo successo dovuto alla bravura dell'attore e alla suggestione patetica dell'argomento «nero» (del resto sottolineata dall'autore nel confronto con I due gemelli veneziani: "Le crime dans la Comédie de l' 'Homme Prudent' fournit du pathétique qui intéresse et touche...» M, II, I, 247) cfr. N. JONARD, n. 1, p. 641.

24. Il racconto delle incontenibili risa degli attori e dell'autore alle prove si legge in M, II, I, 244. Su questa scena si è soffermato Franco Vazzoler, che l'ha contrapposta, per l'intima solidarietà che lega tutti i partecipanti a quella prova, all'atmosfera, che lo studioso giudica —un po' troppo severamente- «didactique, sérieux [...] lucide, froid, livide et lugubre du 'Teatro comico'" (Dramaturgie de l'auteur..., cit., p. 59). 
dell'azione; spiegare i rapporti affettivi e quelli economici; è lui che si definisce reiteratamente secondo la virtù di cui il titolo lo fregia, e infine svolge una vera arringa dove agli argonemti strettamente giudiziali si sostituisce un'eloquenza diretta alla mozione degli affetti. L'impatto doveva essere forte sul pubblico; mentre il commediografo dava prova di qualche disinvoltura morale, nonostante i propositi più tardi esibiti, di fronte all'ambiguità della situazione:

... azonzendo alle rason più sode e più vere le lagreme più calde e più vive, cavae dal fondo del cuor, prostrà ai piè de sto Tribunal, domando e giustizia e pietà: giustizia per do poveri innocenti falsamente accusai; pietà per un povero vecchio, ferio nella parte più delicata, che xe l'onor. La giustizia li assolve, la pietà me consola; e se la giustizia dovesse ancora sospender la grazia, la pietà sia quella che me conceda un'anticipata consolazion. (III, 19; II, $315-316)^{25}$

La sensibilità dell'uomo di spettacolo si manifestò dunque fin dagli esordi nella "tattica» di Goldoni, intento a legare a sé gli attori, fornendo loro gli strumenti capaci di conquistare il pubblico e dunque di assicurargli il successo. Scorrendo la successione dei titoli si rileva una cura precisa tanto nell'alternanza del patetico — che Goldoni definiva interessante- col ridicolo, col brillante, come nell'assegnazione all'uno o all'altro degli attori del ruolo protagonistico.

Volendo soddisfare compiutamente le aspirazioni al successo di D’Arbes, cui riconosceva il merito di averlo sollecitato e convinto ad entrare nella professione di autore di una compagnia, il commediografo trovò, ispirandosi alle doti naturali, ai comportamenti spontanei dell'uomo, il soggetto di una com-

25. L'ampia scena occupa le p. 311-318. Alle capacità attoriali di D'Arbes Goldoni affidava i rimproveri al figlio Lelio (I, 16, 269-270); i numerosi soliloqui (II, 4, 274 e II, 6, 277; II, 14, 288; II, 20, 295-296, che sembra dover qualcosa anche alla tradizione melodrammatica; III, 6, 303, dove Pantalone appare in preda al dilemma; III, 12, 307, vero lamento sull'onore perduto). Forse si deve all'influsso melodrammatico l'idea del lieto fine fondato sul contagio espansivo della clemenza generosa, argomento dell'allocuzione di Pantalone nell'ultima scena; all'attore Goldoni affidava una battuta in cui si consolava degli effetti positivi della propria prudenza, ricorrendo a una metafora nautica, notoriamente topica per le melodrammatiche arie di paragone: la prudenza l'avrebbe guidato al porto della felicità, nonostante le tempeste e gli scogli (cfr. p. 322-323). Sulla commedia cfr. Guido NICASTRO, Dalla commedia dell'arte alla commedia di «carattere»: litinerario di Carlo Goldoni dall' "Uomo di mondo" all" "Uomo prudente», in «Studi goldoniani», n. 6, 1982, p. 131-163. In particolare lo studioso nota ancora nelle prime commedie, più tardi rielaborate per la stampa con sovrapposizioni moralistico-ideologiche, un movimento «dal 'teatro' al 'mondo'", cioè l'intrusione di un "carattere» (il «mondo») nell'organismo dello scenario, cioè il «teatro" (p. 163); cfr. anche Piermario VesCovo, La meccanica e il vizio, in margine all" "Uomo prudente», in "Annali di Italianistica», XI, 1993, numero monografico Goldoni 1993, p. 183-203. 
media ridicola, I due gemelli veneziani, ${ }^{26}$ decisamente alternativa alla precedente esibizione patetica. L'attore, senza maschera, avrebbe dovuto mettere a profitto le proprie risorse mimiche e le caratteristiche fisionomico-comportamentali, quali si erano rivelate all'attento scrutatore:

Tantôt c'étoit l'homme du monde le plus riant, le plus brillant, le plus vif; tantôt il prenoit l'air, les traits, les propos d'un niais, d'un balourd, et ces changemens se faisoient en lui tout naturellement, et sans y penser. (M, II, I, 246)

Dunque queste reminiscenze, per quanto tardive, ribadiscono l'importanza di una drammaturgia fondata sull'attore; più puntualmente si nota che, nell'Autore a chi legge, il libro del teatro è rievocato nella duplicità del suo magistero: l'esempio dei classici, a partire dai citati Menaechmi plautini, rappresenta l'idea del teatro inteso come tradizione letteraria; ma le è subito accostata l'altra idea del libro del teatro, costituito dalla intelligenza unita alla fisicità degli interpreti:

... certa scienza ch'io aveva della straordinaria abilità del bravo Comico [...] nel fare il diverso Personaggio dello spiritoso e dello sciocco ... (A, II, 155)

Il virtuosismo attorico di D’Arbes dovette risplendere nei reiterati equivoci, nella sapienza dei passaggi dall'uno all'altro dei gemelli, fino al culmine della morte in scena dello sciocco Zanetto che sorbiva volontariamente il veleno. Un tale esito contraddiceva, persino sfacciatamente, la convenzione comica e avrebbe potuto sorprendere sgradevolmente gli spettatori. Invece l'autore aveva tanto giustamente valutato la capacità dell'esecutore, che quell'evento insolitamente infausto

non reca all'uditore tristezza alcuna; ma lo diverte per la sciocchezza ridicola, con cui va morendo il povero sventurato. Io non credo arrogante la mia franca asserzione [che quella commedia potesse 'passare per buona'], quando ricordomi delle risa da cui si smascellavano gli spettatori universalmente, sul momento delle sue agonie e de' suoi ultimi respiri. (ivi, 156)

26. La commedia, composta a Pisa nel 1745, fu rappresentata lo stesso anno a Livorno, poi a Lucca nel 1746 e a Venezia nel 1748. Quanto al rapporto tra autore e attore è utile rileggere la prefazione al Frappatore, già Tonin Bellagrazia, del cui insuccesso si è già detto, e che Goldoni dice di aver composta per compiacere "un bravo comico", cioè D’Arbes: "Questa posso dir che sia stata la Commedia fatale che mi ha nuovamente tentato per il Teatro, dopo che avea risolto di abbandonarlo, e che stavami in Pisa ad esercitare l'avvocatura». In questa prefazione Goldoni ha raccontato per la prima volta la visita dell'attore nel suo studio pisano, riferendone il gesto di «battersi colle mani la sua pingue rotonda pancia, dimenare graziosamente il capo» e il «bellissimo lazzo comico» con cui gli aveva anticipato la retribuzione. (A, II, 95. La prefazione comparve nel t. X dell'ed. Paperini, nel 1757) 
Il commediografo aveva contato sull'efficacia del Pantalone, in condizione di «ostentare» sul proprio volto gli effetti delle differenti passioni: ${ }^{27}$ al tour de force della composizione di una pièce, fondata sull'eguaglianza fisica di due individui, di "carattere» opposto (e la nozione di "carattere» aveva qui rilievo scenico ma non ancora spessore morale), e dunque destinati a comparire alternativamente in scena, corrispondeva esattamente il tour de force dell'attore, capace di mutare subitamente e reiteratamente espressioni, gestualità, insomma "gioco" secondo la migliore tradizione dello studio segondo l'arte, nel cui ambito ancora si muoveva il commediografo. ${ }^{28}$

Né si può trascurare l'impegno esibito dall'autore e dall'attore in direzione patetico-moraleggiante nell'Avvocato veneziano, commedia in cui si coniugavano molteplici interessi e diversi espedienti per la conquista del consenso degli spettatori. Movendo - com'è evidente- dalla propria esperienza, il commediografo sostanziava il realismo dello studio di un ceto sociale e professionale particolare; ma contemporaneamente, giocando sul dilemma tra obblighi deontologici e affinità amorose, sollecitava l'adesione simpatetica del pubblico alle vicende dei protagonisti, nonché dei loro interpreti. A D'Arbes e a Teodora Medebach era riservato il netto predominio della scena, dove essi avrebbero potuto mostrare la propria abilità nell'actio retorica l'uno, l'efficacia convincente nelle scene patetiche entrambi.

Il particolare successo dell'interprete, docile e versatile, giovò all'autore, che crebbe nella considerazione del capocomico. Ma la precarietà della professione spinse il bravo D'Arbes ad accettare le allettanti proposte della corte di Dresda, dove si trasferì, lasciando un penoso quanto pericoloso vuoto nella compagnia. Medebach e Goldoni s'impegnarono nella difficile ricerca di un sostituto, individuando infine un giovane di talento, Antonio Mattiuz, che recitava all'improvviso, sotto lo pseudonimo di Collalto, in «compagnie volanti».

Il rapporto che Goldoni instaurò con quell'attore, dotato di intelligenza ma non di éducation è molto interessante: il commediografo che era stato fin a quel momento osservatore di individui da un lato, dall'altro di personalità professionalmente mature, che si offrivano come «fonti» di invenzioni sceni-

27. A proposito della prima commedia destinata ad un attore privo di maschera - il Golinetti- cfr. M, I, XL, 185. A questo proposito, con la solita finezza Jonard osserva che Goldoni avrebbe esagerato il ruolo «storico» di Golinetti nella realizzazione della Riforma i cui lineamenti erano tutt'altro che definiti. Della commedia dell'arte l'autore avrebbe accettato — a quel tempo - le strutture, sostituendo però alla «rigidité du masque la mobilité d'un personnage copié d'après nature». (n. 1, p. 628)

28. Ancora Jonard nota che "comme précédemment, le succès fut un succès d'acteur. Goldoni le reconnaît d'ailleurs volontiers dans les Mémoires (cfr. I, I, 247) comme dans la préface [cfr. II, 155-156 ...]. Quoi qu'il en soit, on reste encore dans le domaine de la commedia dell'arte» (p. 641). Lo studioso cita qui un luogo significativo di Mario Baratto; questi, parlando dei Due gemelli veneziani, aveva rilevato come vi fossero ingrandite le incoerenze sceniche di quel genere spettacolare, nonché la mancanza di moralità, tutte doti che mettevano a nudo «sa gratuité et le goût d'une société qui l'acclamait». (Les Deux Jumeaux de Venise, in Sur Goldoni, Paris, L’Arche, 1971, p. 155-56) 
che, si dedicò alla formazione di un attore, che si prestava docile e confidente al suo magistero. Si potrebbe quasi supporre che per quell'attore Goldoni abbia messo a punto una pedagogia circa il nuovo metodo di recitare, cioè che abbia trovato un "materiale umano» da educare invece che da «sfruttare». ${ }^{29} \mathrm{Si}$ tocca qui il senso della "reciprocità» della relazione didattica tra autore e attori, di una complessa interazione culturale, alla cui riuscita era indispensabile un'intelligenza accresciuta dall'abbandono di ogni presunzione, da parte di entrambi i protagonisti del rapporto.

$\mathrm{Va}$ tenuta nel debito conto anche la dichiarazione pronunciata per bocca di Orazio alle prese con Tonino-Pantalone nel Teatro comico: vi sono rievocati, in faccia al pubblico, alcuni dei successi del recente passato:

Siamo d'accordo, che questa nostra maniera di recitare esiga maggior fatica e maggior attenzione; ma quanto maggior riputazione ai comici acquista? Ditemi, di grazia, con tutte le commedie dell'arte avreste mai riscosso l'applauso che avete avuto nell'Uomo prudente, nell'Avvocato, nei Due gemelli e in tante altre, nelle quali il poeta si è compiaciuto di preeleggere il Pantalone? (I, 4; II, 1053)

L'attore preoccupato delle difficili innovazioni poteva ben essere il Collalto, ma le parole imposte ad Orazio, erano un memorandum diretto a tutto il teatro: a chi occupava la scena, come alla sala e ancora all'impresario-interprete, costretto a dichiarare pubblicamente l'apprezzamento al suo commediografo, meritevole di aver risollevato le sorti economiche della compagnia, grazie alla scommessa delle sedici commedie nuove, tanto più spericolata in quanto annunciata dopo il "fiasco» dell' Erede fortunata.

Le lezioni dell'autore, commisurate alle doti native dell'attore Collalto, assicurarono un felice esito alle commedie "appoggiate» proprio alle sue mature risorse, riconosciute pubblicamente soprattutto nella prefazione all'Avventuriere onorato (rappresentato nel carnevale 1751) del 1753, dove era motivata la scelta di far parlare in veneziano il giovane protagonista Guglielmo, parte in cui eccelleva:

29. L'importanza della collaborazione si rivela dall'elenco dei titoli per cui Goldoni impiegò il Pantalone Collalto: Le femmine puntigliose (Mantova, 18 aprile 1750); Il Bugiardo (ivi, primavera 1750); L'Adulatore (ivi, primavera 1750); Il Cavaliere di buon gusto (Venezia, autunno 1750); Il giuocatore (Venezia, carnevale 1751); La finta ammalata (ivi, carnevale 1751); Lincognita (ivi, carnevale 1751); La donna volubile (ivi, carnevale 1751); I pettegolezzi delle donne (ivi, febbraio 1751); La Castalda (ivi, autunno 1751); Il Tutore (ivi, autunno 1751); La moglie saggia (ivi, carnevale 1752); Il Feudatario (ivi, carnevale 1752); La serva amorosa (Bologna, primavera 1752); I puntigli domestici (Milano, estate 1752); La figlia obbediente (Venezia, autunno 1752); I mercatanti (ivi, carnevale 1752); Le donne curiose (ivi, carnevale 1753). In altre vesti Collalto recitò Il Poeta fanatico (Tonino, Milano, estate 1950); Il vero amico (Florindo, Venezia, carnevale 1751); L'avventuriere onorato (Guglielmo, ivi, carnevale 1751); Le donne gelose (Baseggio, ivi, carnevale 1752). Nei Mercatanti Collalto aveva interpretato il doppio ruolo del vecchio e del giovane. Per l'edizione fiorentina (Paperini 1757) l'autore predispose un testo destinato ad attori diversi; i personaggi ebbero i nomi di Pancrazio e Giacinto. 
un valente Giovine [...] brillantissimo in tali caratteri veneziani, senza la maschera sostenuti, [questi] mi assicurava di un esito fortunato; lo che difficilmente allora avrei conseguito, se ad altro Comico avessi anche in altro linguaggio una cotal parte addossata. (III, 869) ${ }^{30}$

L'asserzione può trovare conferma quando si consideri la commedia $I l$ vero amico, composta a istanza del Collalto, ${ }^{31}$ desideroso di interpretare una parte di nobile moralità. In verità il lavoro, che fu coronato dal successo di pubblico, sviluppa un intreccio di sapore melodrammatico, nuovamente fondato com'è sul dilemma tra l'amore e l'amicizia, tra la passione e il dovere, cui tutto viene sacrificato nello scioglimento, caratterizzato dalla catarsi ammirativa, tipicamente melodrammatica. L'elemento «romanzesco» - denunciato dall'autore- è costituito da un equivoco, a dire il vero molto sfruttato, fondato su una lettera; espediente reimpiegato anche nell'Avventuriere onorato, dove donna Livia lo utilizza per scoprire finalmente i sentimenti di Guglielmo. L'autore dichiarava la propria predilezione per il soggetto, dove brillava il sacrificio in nome della virtù; perciò egli aveva curato il dialogo nelle scene interessanti, cioè patetiche, in cui aveva messo a confronto prima $\mathrm{i}$ due amici-rivali; poi l'uno - innamorato riamato- posto a fronte della giovane Rosaura, sacrificava le proprie fortune a quelle dell'altro. Proprio in tali circostanze doveva riuscire commovente il "virtuoso» Collalto, cui Goldoni affidò molti personaggi portatori delle proprie idee di onore, di saggezza: i suoi Pantaloni-mercanti borghesi idealizzati. Tali sono, oltre al padre del Bugiardo, il socio del Cavaliere di buon gusto, il Tutore, il tenero e apprensivo padre della Moglie saggia, il sentenzioso personaggio che enuncia la morale nei Puntigli domestici.

Allo stesso attore Goldoni affidò l'esecuzione di un'altra commedia almeno in parte autobiografica, secondo quanto — sia pur con qualche modesta cautela - si legge nella dedica alla Marchesa Lucrezia Bentivoglio Rondinelli:

30. La prefazione del 1753, abbreviata per l'edizione Pasquali del 1762, offriva all'autore l'occasione per lamentare le scorrettezze dell'editore Bettinelli e dell'impresario Medebach, che all'altro forniva i primitivi copioni, come cosa di sua proprietà. Le frequenti rimostranze in merito di Goldoni ne confermano l'autocoscienza gelosa di «scrittore».

31. Così riferisce Giuseppe Ortolani nella nota a p. 1198 del t. III. Jonard ha dedicato a questa commedia una nota molto interessante nel suo commento ai Mémoires: la pièce «se situe bien à l'intérieur d'une tentative de moralisation de la comédie»; il suo successo sarebbe «incompréhensible si l'on ne tient pas compte de l'engouement du public pour les comédies sentimentales et le goût de Goldoni pour les situations pathétiques» (p. 649, a proposito di $\mathrm{M}, \mathrm{II}, \mathrm{X})$. Non si tornerà ora sulla diatriba Goldoni-Diderot a proposito dell'originalità $\mathrm{e}$ paternità di questa commedia. Basti il rinvio alla nota dell'Ortolani (III, 1197-1198) e ai recenti contributi di N. MANGinI, La polemica Goldoni-Diderot, in G. PADOAN (a c. di), Problemi di critica goldoniana, Ravenna, Longo, 1994, p. 261-272; Françoise DeCROISETTE, De la scène à la page, premessa alla trad. franc. del Vero amico, in C. GolDONI, «Les Femmes courieuses» suivi de "Le Véritable Ami», Textes français de Anne Manceron, Françoise Decroisette, Arles, Actes Sud-Papiers, 1996, p. n.n., ma 93-97. 
... non nego [...] che qualche analogia non passi fra il Protagonista e l'Autore. La patria, il genio, le professioni, le persecuzioni medesime del povero mio Guglielmo in me facilmente si potrebbono riscontrare. (III, 865)

Solo speciale stima e affetto per l'attore possono aver indotto il drammaturgo ad affidargli la confessione della delusione, che gli avrebbe inflitto, già in quei primi, "gloriosi» tempi della Riforma, l'atteggiamento del pubblico, il quale gli avrebbe fatto apparire ancor più faticosa la professione di «scrittor di commedie», su cui vertono queste considerazioni amaramente autobiografiche:

Il comporre per i teatri lo chiamano bella professione, mestier dilettevole? Se sapessero tutto, non l'intenderebbono già così. Di quanti esercizi ho fatto, questo è stato il più laborioso, il più difficile, il più tormentoso. Oh, l'è pure la dura cosa faticare, sudare, struggersi ad un tavolino, per far una teatrale composizione, e poi vederla gettar a terra, sentirla criticare, lacerare, e in premio del sudore e della fatica, aver de' rimproveri e de' dispiaceri! [...]poche volte l'universal si contenta, e poi quand'anche siasi più volte di uno scrittor compiaciuto, una cosa sola che sia, o che sembri esser cattiva, fa perdere il merito a tutte le cose che furono applaudite. E se la lode si dà a mezza voce, il biasimo si precipita sonoramente e con baldanza. (L'avventuriere onorato, I, 15; III, 891) ${ }^{32}$

Ma Collalto seppe anche prestarsi alle interpretazioni di vecchi storditi da una tardiva passione amorosa, nella Castalda e nella Serva amorosa; o di giovani momentaneamente traviati, come l'Eugenio della Bottega del caffé, o il Pantaloncino, poi Giacinto dei Mercatanti.

32. Ribadisco che il rammarico esplicito del Goldoni investiva il solo pubblico. Ancora nella prefazione del 1753 (posteriore di due anni alla recita) si legge: «Dicesi che la Compagnia di que' Comici, per la quale incessantemente io scrivo, sia meco in discordia; dicesi perfino l'altissima bestialità, che siam venuti alle mani. Giuro non aver mai avuto che dire con esso loro, anzi non essere io stato mai né più quieto, né più ben veduto dai Comici di quel ch'io sono presentemente. Innamorati delle mie Commedie, le rappresentano con valore, con attenzione, con esemplare rassegnazione, e a confusion de' maligni se ne vedranno gli effetti». (III, 871) Sarà tuttavia bene ricordare che proprio in quello stesso anno si rompeva il sodalizio artistico tra Goldoni e la compagnia Medebach, a quel che pare per l'avarizia di questo. Lamenti per le delusioni procurategli dal pubblico si leggono anche nel volume V dell'edizione Paperini 1753, nella dedica del Giuocatore al Conte Parmenione Trissino. (cfr. III, 487) L'avventuriere onorato nel corso della vicenda è costretto dai vari personaggi che lo riconoscono, a rivelare di aver dovuto esercitare le più diverse professioni, di essere stato di volta in volta maestro, medico, segretario, avvocato, cancelliere criminale. La ricca vedova che ne è invaghita gli consiglia di seguitare a coltivare la professione di scrittore di commedie. La pièce è costruita su una serie di incontri e sull'equivoco del dono da parte dell'innamorata Livia di venti doppie, per il tramite di donna Aurora, sulle cui economie di famiglia grava continuare l'ospitalità del povero Guglielmo. Quel denaro si dimezza, si raddoppia, passando dall'una all'altra mano, ma aiuta l'azione ad approdare al lieto fine delle convenienti nozze dell'avventuriere con la ricca vedova. Non trascurerei tuttavia che il lieto fine «costa» il sacrificio di Eleonora, già fidanzata di Guglielmo ed alla fine costretta a «sublimare» il proprio amore nel sacrificio del ritiro in convento. In questa particolare soluzione si potrebbe ravvisare un ulteriore debito alla tradizione melodrammatica, genere in cui la «disfatta» delle donne è una costante (cfr. C. CLEMENT, L'opéra ou la défaite des femmes, Paris, Grasset, 1979). 
Quest'ultimo lavoro ebbe dapprima - nel 1753-il titolo I due Pantaloni. In un certo senso l'autore ripeteva l'exploit dei Due gemelli veneziani, affidando due parti — questa volta opposte in senso morale- allo stesso interprete, il «volenteroso Pantalone per cui la scrisse», che possedeva «l'abilità di far da vecchio e da giovine eccellentemente». Il tour de force tecnico consisteva ancora nel non far incontrare i due personaggi, mentre il messaggio ideologico era spiegato così:

Pancrazio ci rappresenta un Mercante onorato, di buona fama e d'illibata coscienza, il quale anche in mezzo alle calamità ed ai pericoli, teme di commettere un'azione indegna, approfittando dell'altrui buona fede col pericolo di dover fallire. Questo carattere meriterebbe esser distinto in tele e scolpito in marmi, per regola e buon esempio di chi non ha la fortuna di ben conoscerlo. (A del 1753 per l'edizione Paperini, ora IV, 702) ${ }^{33}$

Si potrebbe persino supporre che il Pantalone Collalto abbia «ispirato» il drammaturgo con la morale serietà della sua persona, più ancora che con le sue risorse fisiche; infatti solo al Tonino del Poeta fanatico ${ }^{34}$ si chiede di can-

33. Goldoni non dimenticò di solleticare la corda patetica dei suoi ascoltatori; fiduciose nell'abilità dell'attore, gli affidò una lunga «tirata» diretta al mercante olandese Rainmere, al quale Pancrazio chiede aiuto nella difficile congiuntura, facendo appello alla propria intemerata fama, e dissociando la propria personalità da quella del figlio discolo, meritevole di condanna, e tuttavia salvato — non si saprà mai se davvero redento- da Giannina, decisa e forte della propria cultura, inusuale per le fanciulle veneziane. (III, 4; IV, 759-60) Nella prefazione al Tutore, un altro personaggio affidato all'interpretazione del Collalto, Goldoni volle spiegare la propria antitradizionalistica poetica comica. Nell'intento di ampliare le risorse del genere spettacolare comico, per il quale si riconobbe più dotato che per quello della tragedia, non si limitò a castigare ridendo mores, ed avocò alla commedia il diritto, la capacità, l’opportunità di esaltare la virtù: «lo scopo mio principalissimo è stato, e sarà sempre mai, di mettere la virtù in prospetto, esaltarla, premiarla; innamorare gli spettatori di essa, e darle poscia maggior risalto col confronto dei vizi e delle loro pessime conseguenze. Ecco dunque con tale idea formato il mio Tutore...». ( IV, 141-142)

34. A Tonino, nel Poeta fanatico, l'autore affidò il compito di delineare i ritratti di due attrici, Caterina Landi, di carattere e fisico opposto rispetto a Teodora: «Ella la xe una signora che obbliga a prima vista, che liga i cuori delle persone, e che imprime in tel medesimo tempo amor, reverenza e respetto"; a lei il personaggio dedicava anche un sonetto: «Morbido e folto crin, fra il biondo e il nero, / Spaziosa fronte, e bianco viso e pieno, / Occhio celeste, or torbido, or sereno; / Angusto labbro, rigoroso, austero./ Tenera e breve man, degna d'impero, / Candido, bipartito, amabil seno, / D'ogni proporzion corpo ripieno, / Aria sprezzante, e portamento altero. / Questa è di voi visibile bellezza, / Ma di gloria maggior degna vi rende / La velata beltà, che più si apprezza. / Spirto, che tutto vede e tutto intende, / Arte, che tutto brama e tutto sprezza, / Cuore, che manda fiamme, e non s'accende». (II, 10; III, 289-290 e 291) La terza donna della compagnia, Vittoria Falchi, Eleonora, sostituta di Teodora quando questa era malata, veniva così descritta, ancora per bocca di Collalto-Tonino: «Ghe dirò: quando m'avesse da innamorar, me piaserave una donna de statura ordenaria, ma più tosto magretta, perché el troppo grasso me stomega. Averia gusto che la fusse brunetta, perché dise el proverbio: El bruno el bel non toglie, anzi accresce le voglie. Voria che la gh'avesse do bei rossi vivi sul viso, la fronte alta e spaziosa, la bocca ridente coi denti bianchi, e sora tutto do bei occhi negri, piccoli e furbi. Una bella vita, un bel portamento, un vestir nobile e de bon gusto, che la parlasse presto e pulito, e che sora tutto la fusse bona, sincera, e affabile, e de bon cuor». (III, 11, 316) 
tare in scena, dunque di profittare del dono "naturale» di una discreta voce. Con vera insistenza, invece, il drammaturgo affidò a questo interprete battute che esprimevano i propri ideali etici, o riflessioni sulla difficile professione mercantile, regolata da norme riconosciute crudeli; si veda l'emblematica confidenza del mercante Pancrazio al proprio servo:

Eh Faccenda mio, questo nostro mestiere è delicato assai. Quello che ci tiene in piedi, è la fede, il credito, l'opinione. Tanti e tanti hanno più debiti di me, e tutti loro credono, perché la fortuna li aiuta, e si mantengono a forza di apparenza. Ma quando un uomo principia a dar indietro, quando principia a mancar di credito, tutti gli sono addosso, tutti cercano di rovinarlo, tutti attendono di godere la bella scena; e sapete perché? Per invidia del bene degli altri, e per amor del proprio interesse. Perché la torta si divida fra di loro, e il precipizio di un pover'uomo accresca i loro utili, moltiplichi loro le corrispondenze, e dia fomento e pascolo alla loro maledetta ambizione. (I Mercatanti, II, 6; IV, 738)

L'attore, duttile e affinato, personalmente addestrato dal commediografo nel suo nuovo metodo di recitare, abile anche nelle parti negative - come interprete, ad esempio, del figlio discolo dell'anziano mercatante - testimonia la felice convergenza delle "virtuose» ambizioni innovative di quei protagonisti del Teatro, che ci è confermata dal comune interesse moralmente riflessivo esercitato sui problemi del Mondo.

\section{La «Mulier patiens»}

Cette Actrice estimable, autant par ses moeurs que par son talent, étoit jeune, jolie et bien faite; [...] Sa douceur naturelle, sa voix touchante, son intelligence, son jeu, la rendoient à mes yeux un objet intéressant, une Actrice estimable, au-dessus de toutes celles que je connoissois. (M, I, LII, 232 e 233)

Attraverso questo ritratto Teodora Raffi Medebach viene incontro al lettore dei Mémoires quasi come, a Livorno, aveva accolto Goldoni nella sua casa, alla sua tavola, per un pranzo tanto familiare quanto improntato a garbata proprietà.

Seguendo un uso non raro nelle sue commedie, l'autore affidò alla voce di D'Arbes, Avvocato veneziano, un altro ritrattino dell'attrice, che ne rivelava la duplice capacità interpretativa, subito sperimentata già a Livorno dallo scrittore, «sensibile» anche alla capacità seduttiva di quell'insieme di patetico e di brillante:

La gh'ha un'idea cussì dolce, un viso cussì ben fatto, una maniera cussì gentil, un certo patetico missià con un poco de furbetto, che xe giusto quel carattere che me pol. (I, 1; II, 715)

Goldoni aveva potuto allora vedere eseguita la sua Donna di garbo (1743), una commedia composta per la spiritosa servetta Baccherini, abile nei trave- 
stimenti, ma tanto sfortunata da morire prima di portare in scena quel lavoro, ispirato alle sue doti sceniche. Il côté «furbetto» resse bene la parte e soddisfece l'autore nella messinscena della compagnia Medebach; ma quel complesso volle rendere compiuto onore al poeta, appena "conquistato" da D'Arbes, mettendone in scena la Griselda, una tragicommedia dedotta dal melodramma di Apostolo Zeno e arricchita - come si è già ricordato- della figura nobilmente virtuosa del padre della povera pastora, elevata ai fasti nobiliari dopo una crudele sequenza di prove. In questo ruolo Goldoni dovette scoprire la più originale e significativa dote dell'attrice: la sua voix touchante dovette parergli straordinariamente efficace nel genere patetico, perché sapeva contagiare agli spettatori le proprie dolorose passioni, certo aiutata dalla sua delicata bellezza, cui le lacrime, attributo specifico della mulier patiens, aggiungevano un fascino indubitabile, tanto che da quel momento il poeta di teatro capì come e quanto avrebbe potuto avvantaggiarsi, ai fini della sua idea di rinnovamento morale e serio della commedia, dell'archetipo melodrammatico della vittima innocente.

Ancora nella Vedova scaltra (1748) Teodora fece valere le capacità di frizzante spirito, tradizionalmente richieste alle servette, e in quell'occasione riservate alla giovane vedova Rosaura desiderosa di conquistare con l'amore, in una giornata di festiva, carnevalesca "vacanza» dal peso degli obblighi, la pienezza della propria femminilità, a lungo e onestamente sacrificata ai doveri verso un coniuge troppo anziano.

Poi invece il commediografo preferì insistere sulle altre doti di Teodora: sulla straordinaria risorsa della voce, capace di commuovere, di coinvolgere nelle tenere passioni il cuore degli spettatori. Ciò che Goldoni forse avvertì nell'interprete della ben poco sua Griselda si fece pienamente luce nelle parti di Bettina, Putta onorata (1748) e Buona moglie (1749).

Alla Medebach il commediografo lasciò un largo dominio vocale nelle due commedie, che volevano essere un omaggio a Venezia, di cui si onorava il ceto popolare ${ }^{35}$ attraverso la tenace virtù della protagonista, decisa a resistere ai tentativi di seduzione di un nobile libertino, che la fa rapire e dalla cui violenza la donna si salva a stento.

E' proprio il protagonismo vocale di Bettina che lascia intendere quanto peso abbia avuto nel concepimento e nella conduzione della pièce il fascino della voix touchante di Teodora, sua prima felicissima interprete. Il personaggio - appena evocato prima - si presenta in un suggestivo a solo,

35. Nei Mémoires Goldoni spiega la genesi della Putta onorata come contrepoison ai possibili danni della commedia popolare veneziana Le Putte de Castello, che riscuoteva gran successo, ma a lui pareva pericolosa per i cattivi caratteri posti in scena, per l'intreccio e per la conduzione di questo. Persuaso che il pubblico avesse cominciato a maturare un senso critico che gli consentiva di «préférer la Comédie à la farce, et la décence à la scurrilité pour empêcher le mal que cette Piece auroit pu faire dans les esprits encore chancelans, j'en donnai une dans le même genre, mais honnête et instructive...». (M, II, 251) 
culminante nel pensiero amoroso per Pasqualino, sullo sfondo dell'altana, ${ }^{36}$ dove la fanciulla — povera e orfana - esce a godere il sole, sfuggendo alla costrizione soffocante delle pareti domestiche, della stretta calle: non a caso si può parlare di una parentela della commedia col melodramma, ${ }^{37}$ vista la frequenza con cui la protagonista è posta a confronto, uno per volta, con ciascuno degli altri personaggi: dal dialogo amoroso con Pasqualino, dominato dalle parole tematiche onor e reputazion (I, 6); al contrasto con la sorella Catte lavandera, cui la miseria ha attutito la sensibilità morale (I, 8); al dialogo con Pantalone, occasione per una felice esposizione dell'antefatto, nonché per un confronto energico tra il protettore - la cui benevolenza appare un poco "pelosa»- e la giovane, che protesta tanto la costanza amorosa quanto il tenace attaccamento al valore dell'onestà. Se l'autopresentazione della protagonista può assimilarsi all'aria di uscita di un soprano, il monologo risentito che la oppone al protervo Marchese pare un'aria di sdegno (cfr.I, 13, 444); il soliloquio della scena sedicesima potrebbe assomigliarsi ad un effusivo sfogo emotivo, alla contemplazione della sfortunata condizione di una putta onorata, così come patetica è la battuta, fitta di desolate domande retoriche, della povera rapita, che tuttavia poi esplode in combattive minacce, quasi aria infuriata; e non manca nemmeno l'aria di paragone, quantunque il solenne, tragico mare tempestoso sia qui mutato nel solitario e sconosciuto canal, funestato dalla bissabova. (II, 13, 470)

La seconda pièce del dittico, profittando della precedente esperienza, imponeva alla voix touchante di Teodora di informare il pubblico dell'infelicità di Bettina, sposa di un Pasqualino guastato dalle cattive pratiche, ed ora mostrata nell'atto di cullare il figlioletto. Dopo un breve diverbio colla ser-

36. La stessa altana è rievocata nostalgicamente, associata alla memoria della madre morta troppo presto, in un'ampia battuta rivolta a Catte nella seconda parte della dilogia: «Co me recordo co giera viva mia mare, povereta, che ani che giera queli! Che spasso che aveva su quel'altana! [...] Adesso, tiolè, son qua, povereta, abandonada da tuti. El mario no me vol più ben, el missier no me vien più a trovar; me destruzo in lagreme, e no gh' è nissun che me compatissa». (La buona moglie, II, 5; II, 601; ho segnato in corsivo la citazione del tema delle lacrime)

37. Vi ha fatto cenno Franco FIDO, Prima della "Riforma»: ipernaturalismo e sapienza drammaturgica della "Putta onorata", in ID., Le Muse perdute e ritrovate. Il divenire dei generi letterari fra Sette e Ottocento, Firenze, Vallecchi, 1989, p. 71-91. Le zuffe tra i gondolieri, o i loro canti, sono paragonabili a cori, dei quali l'autore sottolineava con compiacimento il realismo mimetico (cfr. Lettera dell'autore al Bettinelli. Scrittagli l'anno 1751 da Turino, mandandogli la presente Commedia, II, 421-22 e M, II,II, 253), e che lo studioso chiama les cris de Venise. (p. 82) Le voci di Bettina e di Catte, sua sorella, sono definite, rispettivamente, di «soprano lirico o leggero» e di «contralto». (p. 83) I frequenti dialoghi sono detti duetti (p. 75), pur pronti a trasformarsi in duelli. Anche Franco Vazzoler aveva già sottolineato le qualità speciali del personaggio di popolana affidato a Teodora: «il personaggio di Bettina è stato costruito [...] ponendola su un piedistallo eroico-melodrammatico», offrendole battute di registro alto: 'morir più tosto, ma se salva l'onor' (II,10; II, 467), il cui senso era ripreso nel sonetto di congedo: 'piuttosto che far mal, si ha da morir'. (Goldoni, Teodora Medebach, Bettina, in La Putta Onorata, storia di Bettina prima parte, Genova, Teatro di Genova editore, 1987, p. 54-64, la citazione è a p. 58) 
vetta, ancora la protagonista lamentava la propria sorte e rievocava la madre perduta, inframezzando le espressioni patetiche ad altre di risentimento verso la ragazzetta svogliata e malaccorta, sua unica compagna e interlocutrice; seguiva il duetto con la sorella Catte nelle prime scene di realistico, povero interno domestico. A questo si contrappongono, secondo la regola sempre seguita dall'accorto commediografo per ottenere effetto di risalto, gli interni aristocratici, dove la superbia sopravvive alla penuria, provocata dalla dissipazione, dal gioco. Né il Marchese ha ancora deposto le mire sulla povera Bettina, ${ }^{38}$ la quale invece protesta reiteratamente la propria virtù di bona mugier, ad onta delle malefatte dello sposo, cui serba intatto il proprio amore anche di fronte al suocero Pantalone. Due soliloqui che concludono gli atti I e II potrebbero parere arie di entrata, effusioni di tenere speranze amorose rafforzate dall'invocazione al cielo prima; poi di emotiva confidenza diretta al pubblico («Imparé, pute...»; "Vardé...») circa le proprie sofferenze di sposa amante ma negletta (II, 26; II, 592-593); né si potrà trascurare l'impatto emotivo che Goldoni raccomandava alla scena madre del duetto in cui Bettina dà prova dell'estrema dedizione allo sposo, immeritevole, cui fa dono dei suoi manini. (II, 22, 586-589)

La prefazione della Buona moglie rivelava con molta energia gli aspetti più significativi della commedia, evidenziandone la persistente doppia polarità del comico e del patetico, tuttavia pendente verso il secondo termine più di quanto non fosse accaduto nella precedente pièce:

... gli affetti che in essa vengono maneggiati, hanno minor forza quanto al ridicolo; ma quanto alla passione sono più veementi [...] E certamente, se verun'altra mia rappresentazione ha avuto forza di commovere, è stata la presente Commedia; perché quasi esempio di cose vere, ha prodotto sull'animo di qualche uditore mirabile effetto. (A, II, 523) ${ }^{39}$

Non v'è dubbio che ad ispirare il tono appassionato del dittico sia stato il desiderio di valorizzare le doti «vocali» di Teodora; ma anche D'Arbes seppe contribuire, con le proprie capacità di empatico coinvolgimento, a rivelare

38. Una battuta attribuita al Marchese, diretta a Catte, potrebbe suonare come un cenno, che gioca sul contrasto, alla grazia di Teodora resa fragile (ma non per questo priva di seduzione) dalla cattiva salute e dal precario equilibrio nervoso: "Avete un certo brio vivo e disinvolto, che mi va a genio. Vostra sorella è bella, ma è una bellezza troppo malinconica...». (La buona moglie, II, 13; II, 577; corsivo mio)

39. Questa piccola prefazione è una dichiarazione di poetica particolarmente lucida: l'autore vi pone a confronto gli effetti prevedibilmente diversi del proprio lavoro sulla scena e sulla pagina, concludendo che nel ridicolo ha maggiore peso l'apporto degli attori, poiché i lettori non potranno integrare, nel loro spettacolo mentale, le picciole occasioncelle prodotte dai periti Recitanti. Per contro le gagliarde passioni conserverebbero la loro efficacia sulla pagina scritta, la quale saprebbe produrre nel lettore «impressione [...] ed ecciterebbe in lui quelle agitazioni, che negl'introdotti Personaggi si leggono» (A della Buona moglie, II, 523). Nei Mémoires invece l'autore difende la propria innovativa scelta della protagonista nella «classe» dei «virtuosi», anziché in quella dei «ridicoli». (cfr. M, II, III, 256) 
alcune felicissime occasioni: forse suggerì a Goldoni la tenerezza del Pantalone, pronto ad accogliere la nuora, poi intento a bamboleggiare il suo Pantalonzin (La buona moglie, I, 17 e 18; II, 553-56 e 556-557); e ancora la commovente scena della amorevole correzione del giovane scavezzo e il seguente monologo del padre. (cfr. II, 15, 567-569)

Di sicuro significato è anche la scelta di riprendere i personaggi della prima commedia per riproporne la «storia» in un più complesso svolgimento, dove il tempo trascorso e le azioni consumate hanno lasciato le loro tracce: la maturazione di Bettina è un segno della stima dell'autore per le capacità attoriali dell'interprete: sensibile, duttile, versatile, Teodora poté assumere il ruolo e compierlo grazie alla ricchezza della propria personalità. Ed un discorso analogo potrebbe farsi anche per il Pantalone D'Arbes, che ancora conservava qualche tratto moralmente dubbio della maschera primitiva nella prima parte della dilogia, ma mostrava d'aver sublimato in disinteressata, paterna generosità l'antica attrazione amorosa per la nuora. ${ }^{40}$

Un ultimo dato resta da osservare a proposito del «clima» melodrammatico della seconda pièce: le scene conclusive costituiscono un lieto fine improntato al contagio della benevolenza, secondo la catarsi ammirativa più peculiarmente melodrammatica. Si nota un climax ascendente, una gara generosa verso il perdono che redime: Pantalone impetra dalla Marchesa il perdono per il fedifrago consorte, ma vorrebbe mantenere verso il proprio figlio una severità motivata dal pessimismo. A placarlo pensano le preghiere, le rassicurazioni e le lacrime — ancora- con cui Teodora-Bettina sapeva muovere a compassione il personaggio in scena, nonché gli spettatori in sala.

Niora cara, le vostre lagreme m'ha mosso a compassion, ma più m'ha mosso sta povera inocente creatura, che ghe vôi tanto ben. (III, 21; II, 617; corsivo mio)

Persino l'aristocratica Beatrice viene coinvolta nel pathos di quella famigliola e versa anche lei le sue lacrime "per tenerezza».

La vicenda della virtuosa Bettina segna un'importante evoluzione nel teatro di Goldoni e della sua interprete, per qualche anno prediletta. Salvo

40. Nel suo commento ai Mémoires Jonard ha messo in luce l'importanza dell'evoluzione del Pantalone nel dittico goldoniano, dove egli "pour la première fois, acquiert une dignité inaccoutumée. Il y avait déjà eu, il est vrai, le protagoniste de L'uomo prudente, mais il n'avait pas, comme ici, 'l'art de remuer les passions, et de toucher les coeurs jusqu'aux larmes'. C'est dire l'intérêt de cette comédie sérieuse qui s'ouvre sur un intérieur bourgeois à la Greuze où la présence d'un enfant apporte une note d'intimité bien faite pour exprimer la tiédeur du foyer conjugal». (op. cit., n. 3, p. 644) Dello stesso autore, a proposito della poetica del mélange tra i generi, che Goldoni inaugura nel dittico di Bettina, cfr. La nature du comique dans le théâtre de Goldoni, in Le théatre italien et l'Europe (XVII-XVIII siècles). Actes du 2o Congrès International, Firenze, Olschki, 1955, p. 201-214. 
qualche eccezione $^{41}$ i personaggi appoggiati a Teodora dovevano conquistare l'uditorio con le loro virtù, con la loro capacità di sacrificio, fino alla sublimazione, con l'abilità nell'ispirare un'empatica compassione. L'effetto di queste scelte influiva in modo decisivo sull'orientamento della poetica comica, non più ridotta alla morale correttiva attraverso la derisione del vizio, ma ampliata all'esaltazione di una virtù, non "tragica», mediana, ma sicuramente seria.

All'esperienza larmoyante della dilogia popolare si collega l'altro trionfo patetico di Teodora: La Pamela (1750), la cui protagonista intrattiene qualche legame sociale — almeno apparente — con la sua antecedente: povera popolana l'una, serva l'altra, ignara della propria appartenenza al ceto nobiliare. Vi ha fatto cenno giustamente Ilaria Crotti, dopo aver stabilito il legame di questo gruppo di lavori destinato e ispirato dall'attrice con il "grande codice» di estensione europea, il cui topos di base erano le patetiche lacrime:

Quella Pamela borghese-popolare, trasformazione estrema della figura classica della serva [appariva capace di opporre un proprio codice morale a quello antagonistico aristocratico-libertino]; dotata di una straordinaria vitalità fatica [...] della parola e del dialogo [...] la forza di Pamela risulta tutta orchestrata intorno ad una parola che giunge a dilatarsi in ogni spazio testuale, si tratti di battute di dialogo ampiamente orchestrate, monologhi retoricamente connotati o brevi e lucide sentenze. ${ }^{42}$

Della fonte romanzesca Goldoni avrebbe conservato la centralità della parola dell'eroina, sempre contando sull'efficacia della voce di Teodora, capace di reggere un tale ruolo decisamente «solistico», orientato a sviluppare sia l'introspezione monologante, sia l'efficacia dialettica nelle diverse situazioni di confronto o di contrasto con gli altri personaggi. Fin dalla seconda scena, non appena Madama Jèvre la lascia sola, Pamela, abbandonandosi al pianto, svolge in un ampio soliloquio l'esposizione e dichiara la propria condizione sentimentale, che più puntualmente analizza nella

41. Conserva i tratti brillanti Doralice, figlia di Pantalone nella Famiglia dell'antiquario (1749), la quale, con la sua calma e le sue battute taglienti, riesce ad esasperare l'aristocratica suocera, tien testa a tutti e persino al padre. Donna Rosaura delle Femmine puntigliose (1750) appartiene invece alla classe dei ridicoli e vuol essere specchio della vanità. (cfr. A, II, 1120)

42. Ilaria Crotti, Le seduzioni della virtù, Introduzione a C. Goldoni, Pamela fanciulla, Pamela maritata, nell'ed. nazionale delle Opere di Carlo Goldoni, Venezia, Marsilio, 1995, p. 13. La Crotti ha apprestato un utilissimo strumento con la sua edizione; lavoro arduo soprattutto per la complessa vicenda editoriale del testo della fanciulla, di cui non restano manoscritti, ma sul quale Goldoni non si stancò d'intervenire: «Per quanto concerne la prima Pamela, si sono collazionate quelle edizioni settecentesche che in misura maggiore rappresentano le tappe del processo variantistico-editoriale, all'interno di un processo rielaborativo emblematico del fare artistico goldoniano: la princeps Bettinelli, la Paperini, la quinta Bettinelli, la Pasquali ed, infine, la Zatta». («Nota ai testi», ivi, p. 50) 
scena quinta, prendendo spunto dal dono di un anello da parte di Lord Bonfil. ${ }^{43}$

La tessitura discorsiva della pièce si avvale prevalentemente di dialoghi che oppongono a due a due i personaggi, ma consentendo a Pamela altro spazio per squarci «solistici», come accade quando la giovane respinge il tentativo di seduzione del padrone, tanto fermamente quanto abilmente rifuggendo da ogni asprezza: si direbbe che - coerentemente con la poetica esibita dal suo autore - questa Pamela preferisca esaltare le proprie virtù, pur diverse da quelle più rilucenti dei valori sociali del suo allocutario; che pure viene richiamato ai suoi obblighi di classe, ma contemporaneamente al rispetto dell'uguaglianza di tutti gli uomini, almeno sotto il profilo della ragione e dell'amore.

L'onore, già parola tematica nei discorsi di Bettina, torna in quelli di Pamela; anch'ella eroina da melodramma perché «sfortunata», ma tenace custode della propria innocenza, seppure a costo del sacrificio dell'amore; come è dichiarato in un altro «a solo" (I, 17; III, 359). Certo si tratta — vien da pensare - ad una "gestione» assai abile dell'unico patrimonio di una povera ragazza che, forte della propria dignità, — ma anche della presenza del padrone sotto lo stesso tetto - tien testa bravamente all'aristocratico viaggiatore Ernold, che mostra di non aver assolutamente profittato delle esperienze umane conseguibili attraverso la conoscenza del Mondo. (cfr. II, 14, 386-387)

L'onore tenacemente preservato e le lacrime sparse in copia nel corso di tutta la commedia, divengono stimoli efficacissimi (ma quanto consapevoli?) per attizzare la passione di Lord Bonfil, che spiega al cognato — Lord Artur di sentire opportunamente grossolano- in che consista l'irresistibile fascino della giovane:

In una estraordinaria virtù, in una illibata onestà, in un'ammirabile delicatezza d'onore. (II, 2; III, 365)

E alla stessa dichiara:

La tua bellezza è stata creata dal cielo per felicitare un qualche avventurato mortale. (II, 5, 373)

43. Si è voluto rilevare la stretta parentela della tessitura di questa prima Pamela con il genere melodramma, del quale ricorrono i segnali demarcatori artistici e situazionali più consueti: l'esibizione delle lacrime e l'appello alla virtù da un lato, dall'altro alla compassione; la contemplazione lirica e autocommiserante della propria situazione affettiva e sociale, che implica per la ragazza e per il nobile innamorato il dilemma tra passione e dovere; le invocazioni a Dio, le proteste contro la sorte, i numi, la fortuna. Nel n. 8, 1988 degli «Studi Goldoniani» sono comparsi due saggi che insistono sulle situazioni e gli stilemi melodrammatici della Pamela: Alberto BenISCELli, Forza e delicatezza delle passioni. Le metamorfosi di Pamela, p. 85-105; Annamaria FineTTO, La «Pamela» e "La buona figliola»: il linguaggio poetico di Goldoni, p. 107-136. 
Come è noto Goldoni preferì rispettare le convenzioni insieme sociali e teatrali della sua patria, rinunciando alla virtuosa mésalliance, obiettivo dello scrittore inglese e cara a quegli stranieri spiriti illuminati, e spiegò estesamente le ragioni che l'avevano indotto a ricorrere all'abusato espediente dell'agnizione. Dopo tale evento risolutore l'amore reciproco diviene accettabile e legittimato dalle imminenti nozze, sì che gli innamorati possono intrecciare due battute che suonano come un vero duetto, in cui si esalta il reciproco, virtuoso dono del cuore da parte dei protagonisti. Ma Goldoni, dopo aver costretto la sua eroina, e la sua attrice, a versare veri torrenti di lacrime, le attribù̀ poi una chiusa trionfalmente vittoriosa all'insegna della dignità, che rinuncia persino alla superbia di una nascita aristocratica, per rivendicare il pregio della virtù «sulla scena del mondo», come sarebbe convenuto ad un personaggio da melodramma:

Apprenda il mondo, che la Virtù non perisce; ch'ella combatte e si affanna, ma finalmente abbatte, e vince, e gloriosamente trionfa. ( III, 409)

Per qualche stagione il commediografo si compiacque di felicitare di finali trionfi la propria interprete, esemplare nel ruolo di mulier patiens, sfruttandone le doti naturali positive e persino quell'ombra malinconica che la malferma salute gettava sulle grazie di Teodora, senza spegnerle, ma rendendo ben verosimili i personaggi ostinatamente perseguitati dalla sorte e dall'umana cattiveria.

Ancora la Eleonora del Cavaliere e la Dama (1750) trionferà — ma dopo quante lacrime! - dell'ostinata sfortuna, che la riduce nobile ma povera; lontana dal marito esiliato, malato e poi morto; innamorata di un degno cavaliere, ma osservante della fedeltà; ${ }^{44}$ finché la vedovanza le permetterà — dopo conveniente lutto- di attingere a una rinnovata serenità coniugale. Nel clima patetico è coinvolto, anche in quest'occasione, il buon vecchio Pantalone, disinteressatamente soccorrevole verso la gentildonna. ${ }^{45}$

Altri personaggi commoventi per la loro virtù, resistente agli insulti della sorte, o alle difficoltà dei rapporti sociali, sono Vittoria, moglie dello sviato borghese Eugenio, nella Bottega del caffe; Eularia della Dama prudente, commedia in cui Goldoni affida ancora una volta alla Medebach il compito di

44. Rievocando la commedia nei Mémoires Goldoni affermava di aver voluto tracciare una satira del cicisbeismo per lo sbieco dell'elogio della Dama virtuosa e del compassionevole Cavaliere, "qui font contraste avec les ridicules» (M, II, IV, 259). Nell'Autore a chi legge, premessa alla stampa della commedia, nel t. II dell'edizione Paperini (1753), si spiega che l'eliminazione delle maschere, nella rielaborazione del testo, era frutto dell'esperienza - confermata dalle recite fiorentine- secondo le quali al riso non era indispensabile la "caricatura dei volti e dell'abito». (II, p. 627)

45. Jonard vede in questa figura un preciso legame con l'esperienza delle commedie di Bettina e, superando la consolidata lettura sociologica, in chiave marxista, scorge nel mercante di buon cuore il continuatore di una cultura della beneficenza, già proposta da Addison nel personaggio di Freeport, e dalle commedie sentimentali inglesi. (cfr. op. cit. n.1, p. 644) 
fare antitradizionalmente della commedia il genere in cui si propongono pregi da imitare, anziché vizi da rifuggire. La prospettiva consueta avrebbe posto al centro della vicenda la passione della gelosia, da cui don Roberto era affetto, e che provocava il disagio della moglie. Invece proprio a questa fu affidato il ruolo di protagonista, con un rovesciamento che appoggiava al professionismo consumato dell'attrice il compito di far accettare quel nuovo modo d'insegnamento morale in scena: evitando che un ridicolo sconveniente cadesse sull'aristocratico geloso, mentre la nobildonna faceva risplendere la propria prudenza fino al sacrificio, conquistando all'autore il consenso del ceto dominante.

La prefazione è datata 1754 e spiega molto bene le ragioni sociali che presiedettero all'orientamento impresso dall'autore alla sua invenzione: infatti vi si difende l'itinerario non facile, né rettilineo della satira comica della nobiltà, che pure pareva allo scrittore non solo lecita, ma necessaria al teatro, fatte salve le regole della discrezione. Tuttavia egli aveva ancora una volta preferito piegare al proprio intento la vicenda puntando all'esaltazione dell'imitabile virtù, sì che solo per contrasto riuscisse castigato — non deriso però- il vizio. Allo scopo poteva servire la capacità di Teodora di reggere un gioco scenico improntato, questa volta, alla dignità solenne e un poco austera del suo personaggio, non privo di appena percettibili sfumature di compassionevole condiscendenza per il consorte «malato» di gelosia; a favore del quale ella non lesinava la propria sollecitudine attiva e il volontario sacrificio dei pur leciti piaceri delle frequentazioni sociali.

L'archetipo della mulier patiens caratterizzò quasi costantemente i ruoli dell'attrice Teodora, sia pur articolato in una fenomenologia che comprendeva anche una fanciulla pronta ad essere sacrificata dall'improvvida prepotenza del padre, come la più tarda Figlia obbediente di Pantalone (1752) o, come Checchina, che rischiava di rimanere vittima dei Pettegolezzi delle donne (1751).

In quest'ultima pièce Goldoni impiegò, sia pure nella parte secondaria di Catte, Maddalena Marliani, appena ricongiunta, dopo qualche anno di "distrazioni», al marito, il Brighella Giuseppe Marliani da Goldoni apprezzato. Il confronto, destinato a mutarsi ben presto in rivalità nella vita come sulla scena, sarebbe riuscito duro, doloroso per la delicata Teodora, alla quale il commediografo avrebbe assegnato qualche ruolo meno lusinghiero come quelli di Rosaura Finta ammalata e Donna volubile, proposti nella stagione del carnevale 1751.

Nei Mémoires la recita dell'ammalata immaginaria è occasione, per l'anziano scrittore, di lodi dell'interprete, capace di autoironia:

Malgré la simplicité du sujet, cette Piece fut généralement bien reçue, et extrêmement applaudie; elle doit son succès, peut-être, au jeu de l'Actrice qui se plaisoit à se jouer elle-même, et le faisoit sans effort et sans gêne. (M, II, $\mathrm{X}, 287$ ) 
Ma le malattie, gli ostinati «vapori» che avevano afflitto la Medebach avevano perso, nei lontani ricordi, ogni aura di fascinosa malinconia, per presentarsi esclusivamente come fastidioso impaccio al lavoro della troupe. Il commediografo -almeno nelle vesti del memorialista- sembra aver avvertito il disagio proprio in occasione di quel carnevale in cui si trovò guisto a disposizione una Actrice subalterne 46 dotata di un fascino opposto a quello della Medebach. Allora la stella della mulier patiens imboccò la dolorosa parabola discendente, molto evidente nelle pagine dei Mémoires, ma già riscontrabile nella filigrana di alcune tarde commedie del ciclo di Sant'Angelo, quelle incentrate sul ruolo ancora protagonistico della Medebach, cui erano però imposte parti assai meno lusinghiere di quelle del pur recente passato.

Ginette Herry ${ }^{47}$ ha interpretato in chiave freudiana la creazione della Finta ammalata, cogliendovi i segni del disamore di quel Pigmalione verso l'interprete da lui amorosamente formata, ed ora posta in dannoso paragone con la più energica, spregiudicata servetta. A quel punto la persona delicatamente commovente si trasformò in una donna nevrotica, malata di ipocondria, ${ }^{48}$ irritantemente simile al drammaturgo, a sua volta tormentato da consimili crisi ricorrenti:

C'est donc le narcissisme de l'auteur, d'abord flatté puis pris à rebours par la personnalité de son actrice, qui ne peut supporter le reflet de lui-même que la maladie de celle-ci renvoie et qui lui fait construire la suite des pièces de leur collaboration en un cycle cruel dont la courbe, longtemps ascendante, s'inflé-

46. Nella compagnia recitava già una servetta: Colombina; nella Donna volubile Rosaura - la protagonista che il memorialista diceva compiaciuta di imitare in scena una collega, ma di fatto era costretta a mostrare in scena gli aspetti meno lusinghieri del proprio caratterealternava capricciosamente la propria predilezione all'una o all'altra delle due servette, Colombina e la nuova «recluta» Corallina.

47. Ginette Herry ha analizzato con molta finezza i rapporti tra Goldoni e i suoi interpreti; soprattutto la studiosa si è soffermata sulle attrici, di cui il drammaturgo avrebbe saputo scoprire e valorizzare le qualità, al di là degli stereotipi dei loro ruoli fissi: «Libérant ainsi une personnalité ou des potentialités qui dormaient en chacun de ses comédiens préférés, il se fait irrépressiblement, à chaque fois, Pygmalion». (De Teodora..., cit., p. 17)

48. Merita che si citi un passo, pur notissimo, in cui i Mémoires recitano, mescolando le lodi e le recriminazioni: «Madame Medebac étoit une Actrice excellente, très-attachée à sa profession, mais c'étoit une femme à vapeurs; elle étoit souvent malade, souvent elle croyait l'être, et quelquefois elle n'avoit que des vapeurs de commande». In casi simili la minaccia di assegnare "un beau rôle à jouer à une Actrice subalterne» valeva a ridarle la salute. (M, II, X, 286) Ciò sarebbe accaduto anche dopo la sera della prima della Locandiera (S. Stefano 1753), quando Teodora impose fossero sospese le recite del nuovo successo, per sostituirlo con un suo "cavallo di battaglia», La Pamela. La memoria di Goldoni registra un'aspra irritazione: «Ce sont des petites galanteries qui arrivent presque par-tout où le despotisme se moque de la raison. (M, II, XVI, 314, ma per l'insensibilità di Goldoni memorialista ai malesseri di Teodora cfr. anche ivi, 312) 
chit soudain en un scénario de régression jusqu'à l'effondrement dans l'infantilisme. ${ }^{49}$

E quando a Rosaura venne ancora addossata la parte della nobile Moglie saggia, sembrò che l'autore volesse "farle la lezione», imponendole di nuovo il comportamento eroico, facendole esercitare sul palcoscenico una generosità ed una pazienza di cui la donna Teodora non era più capace, davanti all'ascesa irresistibile della rivale. Rosaura recitò la commedia di secondo grado della moglie avvelenata dal marito, incapricciato della cattiva Beatrice; a lui la finta morente offriva un perdono tanto generoso da redimere per contagio l'uomo. Ma l'interprete, consapevole della propria «retrocessione» sulla scena, come nella vita, non avrà certo apprezzato quella prescrizione pedagogica, non priva di crudeltà. 50

Tuttavia poco tempo dopo il commediografo Pigmalione avrebbe offerto all'attrice una sorta di risarcimento almeno morale, nonché artistico. Compreso nel bilancio finale della proficua collaborazione di Goldoni con la troupe Medebach, grazie alla quale era stata realizzata la felice armonia dei rapporti tra l'autore, gli interpreti ed il pubblico, si legge l'addio a Teodora nella prefazione all'ultimo componimento scritto per quegli attori:

... chiamata sulle scene Rosaura, giovane di ottimi costumi, e nei caratteri dolci, amorosi e gentili graziosissima attrice, che ha fatto piangere parecchie volte nella tenerezza delle passioni toccanti il cuore. Devo renderle questa giustizia, d'aver ella con ammirabile prontezza e docilità accolte e bene eseguite le migliori parti delle mie Commedie nel carattere di prima Donna [...] ed in tutte ha sempre fatto grata ed onorevole la sua comparsa; ringraziandola io nuovamente del piacere che dimostrava ella nel recitare le cose mie, e dell'onore che alle medesime ha fatto. (A della Donna vendicativa, IV, 1005)

\section{Un'attrice «vive, spirituelle, et naturellement accorte» (M, II, XIV, 303)}

Alla fine del 1750 la compagnia Medebach si arricchì — lo abbiamo ricordato- di una nuova attrice, Maddalena Marliani che vi assunse il ruolo di

49. Cfr. G.Herry, De Teodora ..., cit., p. 17 e cfr. soprattutto p. 22-26. Di infantilismo avrebbe dovuto far mostra la Rosaura del Contrattempo, una pièce orientata al negativo per il personaggio di Teodora, che potremmo dire grottesco; l'antica, nobile mulier patiens, si balocca con una bambola, come vorrebbe «dans l'impudeur de son désir sans frein, badiner ainsi avec les hommes...». (ivi, p. 25)

50. Jonard precisa che questa commedia, presentata nel gennaio del 1752, segue di oltre un anno il ritorno di Maddalena presso il marito e il suo inserimento nella compagnia; lo studioso ipotizza che lo sviluppo eroico della trama abbia avuto per ispiratrice Nicoletta, paziente moglie del drammaturgo, ma gelosa della nuova donna cui il marito mostrava visibilmente di interessarsi (cfr. la nota a p. 654 della cit. ed. dei Mémoires). Le lodi della saviezza del comportamento di Rosaura contenute nell' "Autore a chi legge», prefazione scritta per la stampa nel vol. IV dell'edizione Paperini, possono suonare come «risarcimento» a Nicoletta; ciò non toglie che si possa pensare ad una lezione imposta alla sicuramente gelosa Teodora, forse in quell'occasione meno disponibile — fuori dalla scena_ al ruolo della mulier patiens. 
servetta col nome di Corallina. La donna era però tanto dotata che presto il commediografo comprese come quel ruolo le si adattasse solo a patto di fargli subire delle profonde trasformazioni, facendo cioè assurgere l'attrice secondaria per definizione - a norma degli statuti dei commedianti- ai ruoli di protagonista. A ben guardare era questo il modo per portare all'ultimo compimento quella "rivoluzione» nel teatro che avrebbe dovuto sconfiggere gli impacci delle convenzioni, delle irragionevoli costrizioni, per lasciare libero il campo ad una sperimentazione volta a far trionfare sia le capacità attoriche dei singoli componenti della troupe, prescindendo dai loro ruoli fissi; sia l'osservazione e lo studio del Mondo, per proporli al pubblico attraverso lo strumento del teatro.

Al tempo stesso la presenza di due attrici dal temperamento, dalle doti umane e di gioco scenico opposte, offriva all'autore un campo di osservazione e di sperimentazione completo, qualora egli avesse saputo sfruttare quella radicale diversità come complementarità. Non è forse necessario sollecitare troppo il testo dei Mémoires per arrivare a tali conclusioni, laddove l'autore rievoca le due personalità, giocando proprio sul contrasto e sulle risorse che ne derivavano a vantaggio di un'immaginazione forse un po' stanca:

Madame Medebac me fournissoit des idées intéressantes, touchantes, ou d'un comique simple et innocent; et Madame Marliani, vive, spirituelle, et naturellement accorte, donnoit un nouvel essor à mon imagination, et m'encourageoit à travailler dans ce genre de Comédies qui demande de la finesse et de l'artifice. (M, II, XIV, 303; corsivo mio)

Costruendo nuove vicende intorno a colei che egli rese la celebre Corallina, Goldoni studiò a fondo un'altra parte dell'universo femminile, approfondendo l'analisi delle condizioni e delle possibilità, delle aspirazioni delle donne. 51

Come a Teodora si addicevano sospiri, lacrime, l'accettazione eroica della propria sorte, e un conseguente comportamento scenico basato sul repos, 52 così la servetta avrebbe conservato una caratteristica del ruolo tradizionale: il

51. Ancora la Herry, movendo dalle storie affidate all'interpretazione di Teodora, ha fatto notare come Goldoni proponesse sulle sue scene «autre chose qu'une bourgeoisie de théâtre et les amours empêchées de jeunes gens de théâtre»; come il tema fosse da lui indagato muovendo dalla prospettiva privilegiata del microcosmo sociale della compagnia per cui e con cui lavorava, arrivando a proporre la donna e la famiglia "comme objet même de réflexion, avec ses déchirures et ses mutations telles qu'elles apparaissent dans la société qui lui est contemporaine». (De Teodora... cit., p. 20-21)

52. A questo proposito cfr. I. CROTTI, "Nota sulla fortuna», in Pamela..., cit., p. 382-383. La studiosa pone in luce l'antitesi del comportamento scenico delle due attrici: alla Medebach si addiceva il repos «almeno in Pamela [...] in quanto centralità di collocazione scenica, che Goldoni espresse esemplarmente, contrapponendo all'andirivieni degli altri personaggi della commedia la radicata e ferma stabilità della figura femminile inglese». Alla sua Corallina — anche in veste di Mirandolina - il commediografo imporrà il mouvement, cioè l'attivismo fattivo. 
movimento, la capacità di trasformazione ora non più tanto esteriore, quanto sviluppata come abilità nel rapportarsi con la sua parola alla misura dell'interlocutore.

Già siora Lugrezia delle Donne gelose mostra la propria irrequietezza mista ad una vera e propria «rivelazione» o "lancio» di un tema, cioè l'ambizione di conservare la libertà conquistata con la vedovanza, mantenuta con l'esercizio di mestieri saggiamente serbati entro il limite di una liceità un poco ambigua. Tale situazione esistenziale è tanto preziosa da non poterla arrischiare porgendo orecchio alle lusinghe dell'amore:

Oe, mi me inzegno: un poco de lotto, un poco de pegni, un poco de noletti... cioè noletti de abiti, intendemose: vôi andar all'opera, vôi andar alla commedia, e no voggio nissun che me comanda [...]I morosi i xe pezo dei marii, i vol comandar a bacchetta, e mi son una testolina che vol far a so modo [...] Rido, godo, me diverto, e no ghe ne penso de nissun una maledetta. (Le donne gelose, I, 12; IV, 379-80)

L'amore geloso della propria indipendenza indurrà Lugrezia a rifiutare l'idea del matrimonio, confidando a siora Giulia: "Oh, maridarme po no! Godo la mia libertà, e me par d'esser una regina». (Le donne gelose, III, ultima, 436)

Goldoni si era forse ispirato all'ansia di indipendenza che aveva spinto Maddalena ad allontanarsi per qualche anno dal marito, e a tornar poi da lui con un piglio di sicurezza che possiamo scorgere nell'accenno di Corallina al proprio imminente insinuarsi nel letto di Brighella, suo marito nella vita. 53

Le donne gelose offrì, a quanto ricordava Goldoni, la prima occasione di rivalità tra la Medebach e la Marliani: il successo di questa fu pieno, la ripagò dell' énergie e della vérité della sua recitazione; mentre l'autore commentò l'evento - dalla parte di Teodora - con una freddezza al limite del cinismo: "Tant pis pour Madame Medebac. La pauvre femme retomba dans ses convulsions». (M, II, XIV, 307)

Le due attrici avevano caratteri, sensibilità e doti attoriche ben differenti, e l'autore spregiudicatamente se ne servì per enfatizzare il gioco dei contrasti, o per realizzare la varietà tonale nella sua produzione, sfuggendo al rischio della noia del pubblico, stuzzicato anzi dalle allusioni alle vicende della piccola e chiusa società degli attori. Certo il commediografo giocò un tiro maligno alla Rosaura Figlia obbediente, ponendo accanto alla patetica protagonista la figura lepida e piacevole di un'ambigua ballerina, Olivetta, accompagnata

53. Cfr. La donna volubile (1751) I, 9; III, 959. La Herry nota, a proposito di questo episodio, che «Le allusioni ai rapporti reali e privati degli attori tra di loro non mancano [...] Il teatro contiene così in filigrana il mondo del teatro, si nutre della vita dell'attore da cui l'autore si aspetta che metta a fuoco addirittura le proprie vicende». (G. HERrY, Goldoni e la Marliani, ossia l'impossibile romanzo, in «Studi Goldoniani», n. 8, 1988, p. 137-158, la citazione è a p. 143) 
dal padre, Brighella, amministratore delle grazie più che dell'arte della figlia. Per i due personaggi "di contorno" l'autore confezionò delle scene tanto godibili che —ad onta della loro riconosciuta superficialità- «Sono riusciti ridicoli per modo questi caratteri, che hanno quasi oscurato il merito della Donna Protagonista». (A della Figlia obbediente, IV, 610)

Intorno al personaggio di Maddalena si direbbe che Goldoni si sia «aggirato» per qualche tempo prima di trovare per lei i ruoli perfettamente adeguati, tanto da assegnarle parti di protagonista, in dispregio aperto alle famose regole dei commedianti.

La Lugrezia delle Donne gelose era ancora prima inter pares, in un lavoro corale; le altre servette cui si è fatto cenno erano figurine appena abbozzate; ma nell'autunno $1751 \mathrm{fu}$ affidato a Corallina il personaggio eponimo della Castalda, posta in una situazione peraltro canonica di "serva padrona», ambiziosa di raggiungere la pienezza del ruolo sociale più lusinghiero. Eppure, nella prefazione, Goldoni spiegò di aver dovuto rifare completamente quella commedia cui era mancato il vero successo, perché egli non «aveva ancor bene il suo carattere [di Corallina] rilevato, come in appresso poi mi riuscì di colpirlo nella Serva amorosa, nella Locandiera...». (A, IV, 7) Certo è che la commedia, nella sua redazione definitiva mostra ed evidenzia il protagonismo di Corallina, che domina anche quantitativamente la scena. 54

54. L'importante prefazione è testimonianza ulteriore dell'autocoscienza del 'letterato'; vi è confermata, come precetto asprissimo, l'intuizione ispirata al giovane dal colloquio con Gaetano Casali. (cfr. PP, XI, già citato infra) L'autore asseriva l'importanza delle figure secondarie; ancora discorreva della diversità che corre tra il testo letterario drammatico e la realizzazione scenica, anche in rapporto all'effetto sul pubblico, che è innegabilmente la vera prova della validità di una pièce. In questa occasione l'autore rivolse consigli e raccomandazioni, circa l'attribuzione dei ruoli, ai dilettanti di teatro, che sempre più frequentemente inscenavano i suoi lavori, e cui egli, nello stamparli, volgeva grati pensieri. Il drammaturgo metteva dunque tutti gli attori in guardia dal pregiudizio circa la "pretensione del primo luogo, onde ne avviene che si rovinano da loro stessi. Ciò accade sovente nelle Compagnie che rappresentano le Commedie stampate. Ciascheduno si crede capace di far la parte migliore, supponendo che questa possa nascondere i suoi difetti. Per me ho sempre detto essere la prima Donna quella che sulla Scena si fa più onore d'un'altra...». (A della Castalda, IV, 7-8) La prefazione comparve nell'edizione Paperini, t. VIII, 1755, per la quale il testo fu rimaneggiato a fondo. Vale anche la pena di comparare i ruoli di Corallina e di Rosaura: nel primo atto la servetta figura in nove scene; le tocca un soliloquio nella scena VI, una piccola arringa a Pantalone nell'VIII e il monologo conclusivo; mentre Rosaura compare in cinque scene. Nel secondo atto prevale leggermente Rosaura, cui spettano sei scene contro le cinque di Corallina, che però chiude l'atto con un monologo di programmatica accortezza economica. Nel terzo atto Corallina domina in otto scene, pronuncia la lunga battuta della scena VII, in cui ricorda pateticamente a Pantalone la propria dedizione, promettendo di fare ammenda dei passati errori «a forza di lagrime e sospiri»; infine ancora lei conclude la commedia celebrando il proprio trionfo nuziale; mentre a Rosaura tocca chiedere aiuto alla serva e nell'ultima scena non ha occasione di parlare. La scena più teatralmente impegnativa addossata alla Marliani che vi dispiegava le proprie attitudini di trasformista è la quinta dell'ultimo atto, nel corso del quale ella, sotto le mentite spoglie del notaio, affrontava Ottavio e gli svelava le male azioni della seconda moglie Beatrice. 
Tale protagonismo si sarebbe consolidato nella Serva amorosa, dove Corallina sarebbe assurta a un ruolo di eroica generosità, dove avrebbe esercitato un dominio vocale della scena, straordinario per una serva. Nell' «Autore a chi legge» Goldoni faceva l'apologia di questa sua scelta, premettendovi il ricordo del successo di pubblico a Bologna, tanto più gradito in quanto contrastante con le tiepide accoglienze riservate alla pièce dai Comici al momento della prima lettura: una tale reazione doveva essere stata frutto dell'invidia dei colleghi e della dolorosa gelosia della Medebach per l'insolito privilegio riservato dall'autore alla Actrice subalterne; fatto che procurò all'opera altre obbiezioni, rintuzzate dall'autore:

Non nego che molto non abbia contribuito all'ottima riuscita di tal Commedia il merito personale di quell' eccellente Attrice, che sostenne mirabilmente il personaggio di Corallina; ma appunto conoscendo io dove potea fare maggior risalto la di lei abilità, ho procurato vestirla d'una prontezza di spirito, che a lei suol essere familiare, e mi è riuscito l'effetto a misura dell'intenzione [...] Dicesi che Corallina parla più che da Serva, ed opera con troppo ingegno e con troppo fina condotta. Ciò è vero, se tutte le Serve hanno ad essere quelle sciocche, che tali Critici avranno praticato sol tanto; [...] Io non imbarazzo questa mia Serva in cose superiori al femminile talento: ella è una femmina più accorta di molte altre, siccome lo è effettivamente l'Attrice medesima, che ha tal carattere rappresentato. (A, IV, 445-446, corsivi miei)

L'autore ribadiva in quest'occasione il proprio principio asprissimo di commisurare le parti alle doti di chi doveva interpretarle; e si trovano infatti nella pagina - datata 1753 - individuati i pregi anzitutto intellettuali della Marliani: la prontezza di spirito, l'accortezza, che nei Mémoires sarebbe stata detta naturale. In conformità all'intelligenza della Marliani, la commedia attribuisce al suo personaggio la capacità di persuadere tutti gli altri a seguire le direttive di comportamento da lei abilmente suggerite, insinuate, infine ordinate.

Dopo essersi incaricata, in un soliloquio con funzione di esposizione, in cui spiega il proprio tenero, fraterno affetto per il signor Florindo (I, 6), Corallina comincia a sollecitare il suo protetto al coraggio, e infatti gli ripete: "Forti [...] forti, coraggio», stimolandolo a non mostrarsi abbattuto a quanti gli vogliono male.

Il compito di artefice del destino altrui è orientato poi su Rosaura, indotta abilmente a credere che Florindo la ami (I, 11). Il commediografo non consente nemmeno che su tale abilità di regista, di burattinaia, si levino eventuali riserve d'ordine morale; perciò offre a Corallina l'opportunità di un articolato soliloquio in chiusa d'atto, nel corso del quale, pur rallegrandosi della bella macchina da lei piantata, la avvenente vedova - a difesa di tutte le donnerivendica il disinteressato amore che la lega a Florindo, cui vuol provvedere per «dolcezza di cuore»: «il mio cuore è di una pasta sì dolce, che chi ne assaggia una volta, non se ne scorda mai più». (I, 14; IV, 477)

Nei confronti di Pantalone, padre di Rosaura, Corallina farà sfoggio di abilità suasoria, prima lusingandone l'orgoglio col lodarne il senno e le 
virtù, poi rivendicando con energia, pronta a sfoderare le armi tipicamente femminili delle unghie, la propria onoratezza contro i maligni mormoratori a proposito dell'ospitalità da lei concessa al ripudiato Florindo. Seguono le più abili insinuazioni, tese a far concedere a Florindo la mano (e la dote) di Rosaura. L'ultima battuta della scena terza del secondo atto è rivelatrice della particolare specie di "amore di benevolenza» che Corallina nutre per il suo fratello di latte: ella confessa a Pantalone di sperare anche in una ricompensa materiale, se la sorte di Florindo volgerà alla prosperità, ma dichiara che nel proprio disinteresse ella è capace di trovare piena autogratificazione:

... quando ancora mi dovessi ingannare, e meco dovesse essere ingrato, non mi pentirò mai di quello che per lui ho fatto, essendo certa e sicura, che il bene è sempre bene; e che tutto il bene, che da noi si fa, viene ricompensato dal cielo; signor sì, dal cielo, che conosce il cuore delle persone, e premia e rimunera le buone opere e le buone intenzioni. (II, 3; IV, 484-485)

Questo è forse il luogo in cui Goldoni concesse il meglio all'attrice che in quel momento possedeva, pare, il suo cuore, e che, per una volta, egli fece capace di un amore di specie tutta particolare, su cui ha richiamato l'attenzione, con molta finezza, Arnaldo Momo. Egli ha riconosciuto in questa Corallina un'altra eroina della libertà, tenacemente difesa anche attraverso l'ossequio alle convenienze sociali, che le garantisce la gestione del proprio spazio intimo, segreto, anzi spirituale; 55 impedendo persino a Florindo di pronunciare al suo indirizzo parole che avrebbero potuto incrinare il delicatissimo edificio di un rapporto amoroso quasi sublimato in materna dedizione, di cui la donna vuol conservare il compiacimento orgoglioso come suprema e segreta ricompensa.56 Situazione infatti ribadita, dopo che Florindo e Rosaura hanno scoperto la reciproca attrazione, abilmente attizzata da colei che rifiuta il titolo di mezzana, inadatto a chi "procura un lecito matrimonio»; quanto a lei, Corallina si proietta orgogliosamente "sulla scena del mondo" - direi da vera eroina, al di sopra del ruolo servile - in un'altra solenne chiusa d'atto:

Si saprà che ho avuto cuore di rinunziare uno sposo civile, un'occasione invidiabile, una grandissima fortuna, per delicatezza d'onore, per zelo di fedeltà, per impegno di vera onestà e disinteressata amicizia. (II, 14; IV, 505)

55. Cfr. A. Момо, La carriera delle maschere,... cit., p. 99 e 100.

56. In tal senso Momo interpreta la lunga battuta che Corallina rivolge a Florindo, con la emblematica chiusa: «vi saró sempre amica, vi saró sempre serva, saró sempre la vostra amorosissima Corallina» (II, 12; IV, 499; cfr. A. МомO, La carriera delle maschere...", cit., p. 101; sul ruolo materno della Serva amorosa, ivi, p. 102). 
Forse la parte della serva amorosa segna l'acme della dignità di MaddalenaCorallina, perché l'autore le concede di amare; la scelta di sposare, invece di Florindo, il buon Brighella è una decisione maturata per proteggere una specie particolare di libertà, diversa da quella perseguita dagli altri personaggi affidati alla stessa attrice: ella vuol salvaguardare quella libertà che Momo ha definito "come creazione e difesa di uno spazio intimo, segreto» 57 , dove la donna può contemplare il proprio "sacrificio» e trarne una soddisfazione a tutti ignota.

L'intellettuale attrice, pur dando prova di saper reggere le sorti di tutti gli altri personaggi, ed anche la propria, si è riservata un angolo di reale affettività; per contro la trionfante Mirandolina, che tuttora occupa nella fama dei posteri il luogo più alto, dovette rinunciare alla dimensione amorosa, di qualsiasi sfumatura; dovette ritrarsene anzi, quasi sgomenta, 58 dopo aver condotto al successo il gioco crudele della seduzione nei confronti del Cavaliere, su cui cade il ridicolo dell'inconsapevolezza.

Nell'impresa la donna — socialmente promossa al ruolo di padronaaveva voluto impegnare tutte le proprie risorse verbali e di azione teatrale: un linguaggio persuasivo, capace di ritorcere contro il misogino le barriere difensive con cui questi si era "catafratto»; una gestualità ed una mimica tanto più seducenti in quanto "contrabbandate» come sincere, spontanee, negate nella loro verità di «recita», e quanto più erano state calcolate e interpretate in modo sapientemente, intellettualmente scevro di ogni empatica compromissione. Questo il disegno dell' accorta Mirandolina, alter ego dell'attrice Maddalena Marliani "pleine d'esprit et de talens» (M, II, XIV, 303); ma il personaggio era stato spinto tanto oltre nel suo gioco da rischiare di compromettervi il proprio autocontrollo razionale.

A quel punto Goldoni induceva la locandiera a ripiegare nuovamente su un matrimonio "conveniente» in tutti i sensi, con Fabrizio. Ma questa volta

57. Ivi, p. 99.

58. Sul personaggio intellettuale di Mirandolina (e sulla sua interprete) ha scritto osservazioni molto fini Mario Baratto. Già nel saggio del 1957 (ma ripubblicato nel volume Tre studi sul teatro, Venezia, Neri Pozza, 1964, cfr. le p. 200-211 dedicate alla Locandiera) lo studioso insisteva sull'elemento della conoscenza del mondo e di sé della donna, che si contrapponeva all'incapacità di autocoscienza del cavaliere, cui non restava che la fuga, come unica via di scampo dalla seduzione femminile. Sulla base della conoscenza di sé, del proprio femminile piacere nell'essere "vagheggiata», Mirandolina poteva costruire la propria recita di secondo grado, provocando sul suo terreno, l'esibito rifiuto dell'amore, il misogino. Alla stessa commedia Baratto ha dedicato la Nota sulla Locandiera (in La letteratura teatrale del Settecento in Italia, a c. di G. Da Pozzo, F. Fido e M. Santagata, Vicenza, Neri Pozza, 1985, p. 125-135), evidenziando la novità di questa pièce, che segna un altro traguardo nello studio della femminilità e in particolare del personaggio della servetta, dall'antica Donna di garbo, alle opere già composte per la Marliani, qui impiegata in un lucido disegno di teatro nel teatro, dove nella conoscenza di sé della protagonista starebbe la sua superiorità sull'antagonista, immeritevole persino della pietà (cfr. p. 128-129). In particolare mi sembra appropriata l'individuazione nel personaggio di una "frigidità d'intellettuale», (p. 130) che non escluderei fosse pure dell'attrice, anche in questo antitetica alla Medebach. 
la donna non intendeva, con quel mezzo, salvaguardare un' intima memoria affettiva: voleva anzi innalzare tra sé e il Mondo - che aveva istituzionalmente libero accesso alla sua locanda, nelle forme più pericolosamente varie- un riparo stabile e funzionale contro i sempre possibili assalti delle passioni. Fabrizio l'avrebbe "protetta» sul doppio versante delle convenienze sociali e delle possibili défaillances personali, assicurandole la programmatica difesa della libertà interiore:

Finalmente con un tal matrimonio posso sperar di mettere al coperto il mio interesse e la mia riputazione, senza pregiudicare alla mia libertà. (La locandiera, III, 13; IV, 848; corsivo mio)

Queste sono le motivazioni della scelta conclusiva dell'itinerario di autocoscienza che anche Mirandolina è "costretta" a compiere attraverso la propria recita, che acquista un valore pedagogico ancor più forte. Esse vanno confrontate con l'asserzione ben diversa che leggiamo all'inizio della commedia, quando l'ipotesi coniugale era stata esclusa proprio a salvaguardia della libertà: 59 intellettualmente raffinata, l'azione della seduttrice non l'ha lasciata uguale a se stessa, quantunque il rischio del coinvolgimento passionale abbia prodotto un movimento psicologico di autodifesa tanto risoluto da rasentare l'autocondanna alla solitudine. ${ }^{60}$ Fabrizio infatti non sarà un compagno alla pari; questo lascia intendere la conclusione della commedia, né oseremo fantasticare di un ipotetico futuro di Mirandolina.

Ebbero invece un futuro, ma non lungo, né armonioso, i rapporti personali ed artistici di Goldoni e di Maddalena. Già al tempo della stesura della Locandiera il commediografo trattava il proprio trasferimento al teatro San Luca; aveva temporeggiato prima di risolversi, trattenuto da "certa mia medesima predilezione», scriveva grato della pazienza ad Antonio Vendramin. ${ }^{61}$ Certo colpisce la condanna moralistica stilata dallo stesso autore pochi mesi dopo la recita, contro il personaggio tanto felicemente delineato, e coronato dal gradimento del pubblico: su Mirandolina sono attirati addirittura i sarcasmi delle "donne che oneste sono [le quali] giubileranno [...] che si smentiscano codeste simulatrici [...] femmine lusinghiere», delle cui arti Goldoni confessava di essere stato vittima, tanto da aver inteso costruire la più morale

59. La decisione di sposare Fabrizio conclude un soliloquio dov'è tracciato il futuro oltre l'azione; essa sembra corrispondere nell'espressione al primo «a solo» di Mirandolina che rifiuta il matrimonio con una sicurezza al limite della superbia: «A maritarmi non ci penso nemmeno; non ho bisogno di nessuno; vivo onestamene, e godo la mia libertà. (I, 9, p. 792; corsivo mio)

60. Baratto fa cenno alla inattingibile solitudine di Mirandolina, sensibile alla fine della pièce (cfr. Nota..., cit., p. 132).

61. Si è pensato che all'esitazione ad abbandonare la compagnia contribuisse l'affezione per la Marliani. Ma la separazione fu provocata dal disgusto per l'avarizia del Medebach. (cfr. la dedica al Vendramin dell'Adulatore, III, 167; eil «Manifesto» per la stampa Paperini, datato 22 aprile 1753, XIV, 87) 
delle sue lezioni teatrali, smascherando e rendendo persino "odioso il carattere delle incantatrici Sirene». (A, IV, 780)

La prefazione è straordinariamente precisa nell'illustrazione degli effetti scenici di taluni episodi, ciò che conferma in modo ben evidente la riuscita dello spettacolo assicurata dall'interprete. Proprio la precisione, artisticamente compiaciuta e insieme moralmente aggressiva, dell'analisi metateatrale fa supporre che sia troppo poco credere dovuti a tardive cautele contro i pedanti rigoristi gli strali intinti d'amarezza contro le arti della seduzione, dell'inganno lusinghiero. ${ }^{62}$ D'altro canto la commedia composta subito dopo, dove la Marliani doveva nuovamente rivestire i panni servili di Corallina, cioè La donna vendicativa, "marchia» la donna insieme con l'attrice, entrambe più che irritate dalla scelta del commediografo di passare ad altro teatro, ad altri attori; ${ }^{63}$ e va ancora notato che nell'Autore a chi legge dell'ultimo lavoro scritto per il Sant'Angelo - vera pagina autobiografica e di storia del teatro- tra le lodi per gli antichi collaboratori, non troviamo accenno a Maddalena, che sembra, anche con tale espediente, verosimilmente identificata nel ruolo basso che avrebbe dovuto assumere. L'autore infatti ricorda che la commedia tenne il cartellone per due sere soltanto:

... non so se arrestato fosse il suo proseguimento da poco applauso, ma posso asserire per la verità, che l'ho veduta egregiamente rappresentare, e la celebre Corallina principalmente ha sostenuto a meraviglia la parte della Vendicativa, quantunque di malanimo lo facesse, e chiedesse al popolo scusa, con un Sonetto non mio, di avere un tal carattere rappresentato, imputando a me la colpa di aver alle Donne appropriato lo spirito della vendetta. $(\mathrm{A}, \mathrm{IV}, 1011)$

La celebre Corallina aveva voluto scrollarsi di dosso la taccia di vendicativa, scusandosi col pubblico; ma anche il commediografo presentò agli spettatori-lettori le proprie scuse motivando la scelta del carattere negativo con le difficoltà della fantasia creatrice, indotta dal troppo lavoro ad attingere alla classe dei viziosi. Il ricorso al complicato congegno, secondo il costume degli Spagnoli, avrebbe dovuto rallegrare il contesto di un intreccio fondato inoltre sull'altro vizio dell'ira; occasione questa offerta ad Ottavio-Medebach, specialista nei caratteri caricati. La Rosaura della Medebach riusciva, senza rinunciare alla propria teatrale bontà amorosa e gentilezza patetica, vincitrice dell'attivismo intrigante della serva Corallina; la quale, avendo già sedotto il vecchio Ottavio, mirava alla conquista del giovane Florindo, tentando di

62. Ancora Ginette Herry, sensibile indagatrice delle vicende delle attrici goldoniane, ha fermato la sua attenzione su Goldoni e la Marliani ... cit., p. 137-158) mettendo a nudo l'intrecciarsi delle vicende personali con quelle artistiche. Cfr. specialmente le p. 154-15.

63. "Coraline très-piquée de me voir partir, et voyant l'inutilité de ses démarches pour m'arrêter, me jura une haine éternelle». (M, II, 16, 316) 
sottrarlo a Rosaura, anche con un complicato imbroglio da lei ordinato con "professionale» spirito di regista. ${ }^{64}$

L'intelligenza —che si direbbe cerebralitá- della Marliani ancora una volta sembra, in quest'occasione, paragonata alla sensibilità tenera di Teodora, quasi «risarcita», sulla scena, delle lacrime che aveva forse versato nella vita, e che avevano scatenato quelle crisi di «vapori», freddamente osservati e poi ricordati dal commediografo. L'accortezza «naturale» di Corallina, subito riconosciuta dall'autore e presto divenuta soggetto e sostegno di intrecci, anche qui regge la trama, la ordisce, le dà ordine, ma senza il successo che aveva coronato i generosi maneggi della Serva amorosa, o il puntiglio della Locandiera: il vecchio già sedotto si riscuote e scompiglia il piano, restituendo la gioia amorosa alla figlia e svergognando l'intrigante cammeriera, ricacciata nella nativa miseria della campagna. ${ }^{65} \mathrm{Il}$ perdono generoso che provoca la resipiscenza finale di Corallina, non attinge la riconquistata armonia «melodrammatica", che coronava le pièces incentrate sull'eroismo patetico di Teodora, o sulla equilibrata, mediana saggezza di Pantalone.

Il colore oscuro del finale «notturno» è il correlativo scenografico, di un trasparente simbolismo, del nero della vendetta. Ma il dominio dei caratteri negativi, che il lieto fine non riesce a controbilanciare, sembra lasciar trasparire la mestizia pessimistica di Goldoni: quell'addio ai compagni di una avventura teatrale vissuta in armonia d'intenti, coronata dal prolungato consenso del pubblico, era segnato da sentimenti più profondi e forse più consapevoli della malinconia di qualunque addio. L'incertezza circa la futura possibilità di far rivivere l'armonioso e virtuoso triangolo era tanto dolorosamente sentita che Goldoni volle esorcizzarla nella premessa alla Commedia, che suona come celebrazione di una vicenda umana e artistica singolarmente positiva.

64. Goldoni mette in bocca a Corallina una battuta in cui è ben spiegato l'inganno da lei ordito, in quella che si potrebbe manzonianamente chiamare la "notte degli imbrogli e de" sotterfugi». (cfr. III, 21; IV, 1073) Di questa commedia ha dato una bella lettura Franco FIDO, Due «notturni»: i commiati di Goldoni dal Teatro Sant'Angelo e dal San Luca, in ID., Il paradiso dei buoni compagni. Capitoli di storia letteraria veneta, Padova, Antenore, 1987, p. 179-194.

65. La Herry ha interpretato questa cacciata dalla città verso i campi come simbolica della «regressione» artistica imposta da Goldoni alla ammaliatrice Sirena, delle cui arti aveva imparato a sue spese a diffidare. Pertanto l'autore «espelle [...] dal suo teatro riformato quella sua servetta, costringendola da se stessa a far ritorno alla commedia dell'arte, anzi ai balli sulla corda...» (Goldoni e la Marliani..., cit., p. 157) 